Pacific

Journal of

Mathematics

TECHNIQUES FOR APPROACHING THE DUAL RAMSEY PROPERTY IN THE PROJECTIVE HIERARCHY

LoRenz HaLbeisen and Benedikt Löwe 


\title{
TECHNIQUES FOR APPROACHING THE DUAL RAMSEY PROPERTY IN THE PROJECTIVE HIERARCHY
}

\author{
Lorenz Halbeisen And Benedikt LÖWe
}

\begin{abstract}
We define the dualizations of objects and concepts which are essential for investigating the Ramsey property in the first levels of the projective hierarchy, prove a forcing equivalence theorem for dual Mathias forcing and dual Laver forcing, and show that the Harrington-Kechris techniques for proving the Ramsey property from determinacy work in the dualized case as well.
\end{abstract}

\section{Introduction.}

Set theory of the reals is a subfield of Mathematical Logic mainly concerned with the interplay between forcing and Descriptive Set Theory. One of the motivations behind Descriptive Set Theory is the strong intuition that simple sets of real numbers should not display irregular behaviour, or, in other words, they should be topologically and measure theoretically nice.

In order to fill this statement with mathematical content, we should make clear what we mean by "simple" and what we mean by "nice". Both questions have a conventional and well known answer:

- The measure of simplicity with which we categorize our sets of reals is the projective hierarchy, in other words, the number of quantifiers necessary to define the sets with a formula in first order analysis (or second order arithmetic).

- A set should be considered "nice" or "regular" if it has the Baire property in all naturally occurring topologies on the real numbers and is a member of all conceivably natural $\sigma$-algebras.

Set theory teaches us that the axioms of ZFC do not entail a formal version of these intuitions: It is consistent with ZFC that there are irregular sets already at the first level of the projective hierarchy. ${ }^{1}$ Thus the focus shifts from proving that all simple sets are nice to investigating the situations under which our intuitions are met by the facts.

\footnotetext{
${ }^{1}$ In Gödel's Constructible Universe $\mathbf{L}$ there is a $\boldsymbol{\Delta}_{2}^{1}$ set which is not Lebesgue measurable and which does not have the Baire property. Worse still, there is an uncountable $\boldsymbol{\Pi}_{1}^{1}$ set with no perfect subset and a $\Pi_{1}^{1}$ set which is not Martin measurable.
} 
A whole array of research in this direction is dealing with the second level of the projective hierarchy. Solovay provided us with the prototype of a characterization theorem for the second level:

Theorem 1.1. The following are equivalent:

(i) Every $\boldsymbol{\Sigma}_{2}^{1}$ set of reals has the Baire property.

(ii) For every real $a \in[\omega]^{\omega}$ the set of Cohen generic reals over the model $\mathbf{L}[a]$ is comeager in the standard topology on the real numbers.

One could call a theorem like this a "transcendence principle over the constructible universe". These principles connect the theory of forcing and the topological properties of the reals. Comparable theorems have been proved in [JuSh89] (for the $\boldsymbol{\Delta}_{2}^{1}$ level) and in [BrLö99] (for different topologies and $\sigma$-algebras).

A particularly interesting instance of niceness in the above sense is the Ramsey property, a topological property which is deeply connected to Ramsey theory and infinitary combinatorics. The Ramsey property is linked to a forcing notion called Mathias forcing, introduced by Mathias in [Mat77], and Judah and Shelah were able to obtain the following Solovaytype characterization for it (cf. [JuSh89, Theorem $2.7 \&$ Theorem 2.8]):

Theorem 1.2. The following are equivalent:

(i) Every $\boldsymbol{\Sigma}_{2}^{1}$ set of reals has the Ramsey property.

(ii) Every $\boldsymbol{\Delta}_{2}^{1}$ set of reals has the Ramsey property.

(iii) For every real $a \in[\omega]^{\omega}$ the set $\left\{r \in[\omega]^{\omega}: r\right.$ is Ramsey over $\left.\mathbf{L}[a]\left[\mathcal{F}^{r}\right]\right\}$ is comeager in the Ellentuck topology. ${ }^{2}$

One connection to Mathias forcing is given by the following result (cf. [HalbJu96, Theorem 4.1]):

Proposition 1.3. If $\mathbf{N}$ is any model of ZFC, then the following are equivalent:

(i) $\mathbf{N}$ is a model in which every $\boldsymbol{\Sigma}_{2}^{1}$ set is Ramsey, and

(ii) $\mathbf{N}$ is $\boldsymbol{\Sigma}_{3}^{1}$-Mathias-absolute. ${ }^{3}$

As the Ramsey property talks about infinite subsets of the natural numbers, it is easily dualized by something we shall call the dual Ramsey property, talking about infinite partitions of the natural numbers. ${ }^{4}$ This

${ }^{2}$ A real $r$ is Ramsey over $\mathbf{L}[a]\left[\mathcal{F}^{r}\right]$ if and only if $\mathcal{F}^{r}:=D_{r} \cap \mathbf{L}[a]\left[D_{r}\right]$ forms an ultrafilter

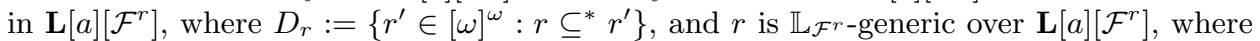
$\mathbb{L}_{\mathcal{F}^{r}}$ is Laver forcing restricted to $\mathcal{F}^{r}$.

${ }^{3}$ Similar characterizations also exist for some other properties, e.g., for Lebesgue measurability and Baire property (cf. [BaJu95, Theorem 9.3.8]).

${ }^{4}$ Infinite subsets can be seen as images of injective functions and infinite partitions can be seen as preimages of surjective functions, so the move from infinite subsets to infinite partitions actually is a dualization process. 
property has been introduced by Carlson and Simpson in [CaSi84] and further investigated in [Halb98-2] and [Halb98-1].

One thing that is striking about the relationship between the Ramsey property and the dual Ramsey property are the distinctive symmetries and asymmetries. This paper can be understood as a catalogue of some of the similarities; in fact, one could see parts of this paper as an attempt to reach the obvious dualization of Theorem 1.2:

Conjecture 1.4. The following are equivalent:

(i) Every $\boldsymbol{\Sigma}_{2}^{1}$ set of reals has the dual Ramsey property.

(ii) Every $\Delta_{2}^{1}$ set of reals has the dual Ramsey property.

(iii) For every real $a \in[\omega]^{\omega}$ the set $\left\{R: R\right.$ is dual Ramsey over $\left.\mathbf{L}[a]\left[D^{R}\right]\right\}$ is comeager in the dual Ellentuck topology.

In order to approach this conjecture and to give an idea what "dual Ramsey over $\mathbf{L}[a]\left[D^{R}\right]$ " could mean, several of the techniques of [JuSh89] and [Mat77] have to be adapted to the new environment:

Mathias forcing has a characteristic product form $\mathbb{M}=\mathcal{P}(\omega) /$ fin $* \mathbb{M}_{\mathbf{U}}$ where $\mathbf{U}$ is the canonical name for the generic ultrafilter added by $\mathcal{P}(\omega) /$ fin. This ultrafilter is in fact a Ramsey ultrafilter, ${ }^{5}$ and Judah and Shelah show in their [JuSh89] that Mathias forcing relative to an ultrafilter is forcingequivalent to Laver forcing relative to the same ultrafilter, provided that the ultrafilter is Ramsey (cf. [JuSh89, Theorem 1.20 (i)]):

Theorem 1.5. Let $\mathcal{F}$ be a Ramsey ultrafilter. Then the forcing notions $\mathbb{L}_{\mathcal{F}}$ and $\mathbb{M}_{\mathcal{F}}$ are equivalent.

This theorem was our motivation to search for a dual version of Laver forcing and the dualization of Ramsey ultrafilters to work towards a dualization of Theorem 1.2.

In our dualized situation there are many things to be done to make sense of the dualized versions: One has to find a dualized version of $\mathcal{P}(\omega) /$ fin and to prove the corresponding product form of dual Mathias forcing (already done in [Halb98-1]), one has to find a dualized version of Ramsey ultrafilters, and one has to make explicit what Laver forcing in this context is supposed to mean.

Section 2 of this paper defines all the dualized notions needed for the technical work on the dual Ramsey property. In Section 3, the reader will find a couple of facts about a dualization of Ramsey ultrafilters; their connection to the game filters from [Halb98-1] is given in the appendix. Section 4

\footnotetext{
${ }^{5}$ A set $\mathcal{F} \subseteq[\omega]^{\omega}$ is a Ramsey filter if $\mathcal{F}$ is a filter and for any colouring $\tau:[\omega]^{n} \rightarrow r+1$ (with $n, r \in \omega$ ) there is an $x \in \mathcal{F}$ such that $\tau \uparrow[x]^{n}$ is constant. Notice that every Ramsey filter is an ultrafilter.
} 
moves on to discuss dual Laver forcing and proves the dualized version of Theorem 1.5.

In Sections 5 and 6 we investigate the extent of sets with the dual Ramsey property in the projective hierarchy. In Section 5 we prove a couple of consistency results for the first three levels of the projective hierarchy. After that, Section 6 looks at the dual Ramsey property from a completely different angle: If we assume an appropriate amount of determinacy, we know that a large collection of sets has the Ramsey property. This result is not at all immediate from the Banach-Mazur game for the topology associated with the Ramsey property. ${ }^{6}$ However, in that section we note that the Harrington-Kechris technique of proving the Ramsey property from standard determinacy (cf. [HarKe81]) alone works for the dualized case as well.

It should be mentioned that the technicalities of the dualization process are not always as easy as they seem in retrospect. Finding the correct and natural dualizations for the interesting notions from the classical case is the most challenging part in this project. After the right dualizations are at hand, in most cases one can follow the classical proofs. So, the merits of this paper lie mainly in the definitions that make the proofs nice and easy and give a proper and firmly rooted understanding of the symmetries. This is also the reason for the unproportional size of Section 2 compared to the other sections.

\section{Definitions and notations.}

2.0. Set-theoretic notation. Most of our set-theoretic notation is standard and can be found in textbooks like [Je78], [Ku83] or [BaJu95]. For the definitions and some basic facts concerning the projective hierarchy we refer the reader to $[\mathbf{K a n 9 4 ,} \S 12]$.

We shall consider the set $[\omega]^{\omega}$ as the set of real numbers. For the Turing join of two reals $x$ and $y$ (i.e., coding two reals into one), we use the standard notation $x \oplus y$.

2.1. Partitions. A set $P \subseteq \mathcal{P}(S)$ is a partition of the set $S$ if $\emptyset \notin A$, $\bigcup P=S$ and for all distinct $p_{1}, p_{2} \in P$ we have $p_{1} \cap p_{2}=\emptyset$. An element of a partition $P$ is also called a block of $P$ and $\operatorname{dom}(P):=\bigcup P$ is called the domain of $P$. A partition $P$ is called infinite, if $|P|$ is infinite, where $|P|$ denotes the cardinality of the set $P$. The equivalence relation on $S$ uniquely determined by a partition $P$ is denoted by $\sim_{P}$.

\footnotetext{
${ }^{6}$ The obstacle is that playing basic open sets in this topology cannot be coded by natural numbers. So the Banach-Mazur games essentially needs determinacy for games with real moves, e.g., $\mathrm{PD}_{\mathbb{R}}$. This is connected to the famous open question whether $A D$ implies that every set has the Ramsey property (cf. [Kan94, Question 27.18]).
} 
Let $P$ and $Q$ be two arbitrary partitions. We say that $P$ is coarser than $Q$ (or that $Q$ is finer than $P$ ) and write $P \sqsubseteq Q$, if for all blocks $p \in P$, the set $p \cap \operatorname{dom}(Q)$ is the union of some sets $q_{i} \cap \operatorname{dom}(P)$, where each $q_{i}$ is a block of $Q$. Let $P \sqcap Q$ be the finest partition which is coarser than $P$ and $Q$ with $\operatorname{dom}(P \sqcap Q)=\operatorname{dom}(P) \cup \operatorname{dom}(Q)$. We say that $P$ is almost coarser than $Q$ and write $P \sqsubseteq^{*} Q$ if there is a partition $R$ such that $\operatorname{dom}(R)$ is finite and $R \sqcap P \sqsubseteq Q$. If $P \sqsubseteq^{*} Q$ and $Q \sqsubseteq^{*} P$, then we write $P \stackrel{*}{=} Q .^{7}$

Let $P$ and $Q$ be two partitions. If for each $p \in P$ there is a $q \in Q$ such that $p=q \cap \operatorname{dom}(P)$, we write $P \preccurlyeq Q$. Note that $P \preccurlyeq Q$ implies $\operatorname{dom}(P) \subseteq \operatorname{dom}(Q)$.

For $x \subseteq \omega$ let $\min (x):=\bigcap x$. If $P$ is a partition with $\operatorname{dom}(P) \subseteq \omega$, then $\operatorname{Min}(P):=\{\min (p): p \in P\}$; and for $n \in \omega, P(n)$ denotes the unique block $p \in P$ such that $|\min (p) \cap \operatorname{Min}(P)|=n+1$.

The set of all infinite partitions of $\omega$ is denoted by $(\omega)^{\omega}$; and the set of all partitions $s$ with $\operatorname{dom}(s) \in \omega$ is denoted by $(\mathbb{N})$.

For $s \in(\mathbb{N})$, let $s^{*}$ denote the partition $s \cup\{\{\operatorname{dom}(s)\}\}$. Notice that $\left|s^{*}\right|=|s|+1$.

For a natural number $n$, let $(\omega)^{n *}$ denote the set of all $u \in(\mathbb{N})$ such that $|u|=n$. Further, for $n \in \omega$ and $X \in(\omega)^{\omega}$ let

$$
(X)^{n *}:=\left\{u \in(\mathbb{N}):|u|=n \wedge u^{*} \sqsubseteq X\right\},
$$

and for $s \in(\mathbb{N})$ such that $|s| \leq n$ and $s \sqsubseteq X$, let

$$
(s, X)^{n *}:=\left\{u \in(\mathbb{N}):|u|=n \wedge s \preccurlyeq u \wedge u^{*} \sqsubseteq X\right\} .
$$

It will be convenient to consider $\omega$ as the partition which contains only singletons, and therefore, for $s \in(\mathbb{N}),(s, \omega)^{n *}:=\{u \in(\mathbb{N}):|u|=n \wedge s \preccurlyeq u\}$.

A family $\mathfrak{F} \subseteq(\omega)^{\omega}$ is called a filter if

$(\alpha) \emptyset \notin \mathfrak{F} ;$

$(\beta)$ If $X \in \mathfrak{F}$ and $X \sqsubseteq Y$, then $Y \in \mathfrak{F}$;

$(\gamma)$ If $X$ and $Y$ belong to $\mathfrak{F}$, then $X \sqcap Y \in \mathfrak{F}$.

Further, we call $\mathfrak{F} \subseteq(\omega)^{\omega}$ an ultrafilter if $\mathfrak{F}$ is a filter which is not properly contained in any filter. Notice that if $X \in \mathfrak{F}$ and $\mathfrak{F} \subseteq(\omega)^{\omega}$ is an ultrafilter, then each $Y \in(\omega)^{\omega}$ with $Y \stackrel{*}{=} X$ belongs to $\mathfrak{F}$, too.

2.2. The dual Ellentuck topology and the dual Ramsey property. Let $X \in(\omega)^{\omega}$ and $s \in(\mathbb{N})$ be such that $s \sqsubseteq X$. Then

$$
(s, X)^{\omega}:=\left\{Y \in(\omega)^{\omega}: s \preccurlyeq Y \sqsubseteq X\right\}
$$

and

$$
(X)^{\omega}:=(\emptyset, X)^{\omega}=\left\{Y \in(\omega)^{\omega}: Y \sqsubseteq X\right\} .
$$

\footnotetext{
${ }^{7}$ We choose this notation because the properties of $\sqsubseteq$ and $\sqcap$ are similar to those of $\subseteq$ and $\cap$.
} 
Obviously, this definition depends on the model we are working in, so, if this should become important, we denote by $(s, X)_{\mathbf{N}}^{\omega}$ the corresponding set interpreted in the model $\mathbf{N}$.

Let the basic open sets on $(\omega)^{\omega}$ be $\emptyset$ and the sets $(s, X)^{\omega}$, where $s$ and $X$ are as above. These sets are called the dual Ellentuck neighbourhoods. The topology induced by the dual Ellentuck neighbourhoods is called the dual Ellentuck topology (cf. [CaSi84]).

A family $A \subseteq(\omega)^{\omega}$ has the dual Ramsey property (or just is dual Ramsey) if and only if there is a partition $X \in(\omega)^{\omega}$ such that either $(X)^{\omega} \subseteq A$ or $(X)^{\omega} \cap A=\emptyset$.

Closely related (but stronger) is the notion of a completely dual Ramsey set: A set $A \subseteq(\omega)^{\omega}$ is said to be completely dual Ramsey if and only if for each dual Ellentuck neighbourhood $(s, X)^{\omega}$ there is a $Y \in(s, X)^{\omega}$ such that $(s, Y)^{\omega} \subseteq A$ or $(s, Y)^{\omega} \cap A=\emptyset$. If we are always in the latter case, then $A$ is called completely dual Ramsey-null. It is not clear if "every projective set is completely dual Ramsey" is really stronger than just "every projective set is dual Ramsey", because we cannot simply translate Lemma 2.1 of [BrLö99], where it is shown among other things that "every projective set is Ramsey" and "every projective set is completely Ramsey" are equivalent.

Carlson and Simpson proved in [CaSi84] that a set $A$ is completely dual Ramsey if and only if $A$ has the Baire property with respect to the dual Ellentuck topology and $A$ is completely dual Ramsey-null if and only if $A$ is meager with respect to the dual Ellentuck topology. ${ }^{8}$ As a matter of fact we like to mention that in the dual Ellentuck topology every meager set is nowhere dense and hence, the dual Ellentuck topology is a Baire topology (i.e., no open set is meager). This corresponds to the similar facts about "being completely Ramsey" and the Ellentuck topology. ${ }^{9}$

2.3. Dual Mathias forcing. The conditions of the dual Mathias forcing $\mathbb{M}^{\star}=\left\langle M^{\star}, \leq\right\rangle$ are the pairs $\langle s, X\rangle$ such that $(s, X)^{\omega}$ is a non-empty dual Ellentuck neighbourhood, and the partial order is defined by

$$
\langle s, X\rangle \leq\langle t, Y\rangle \Leftrightarrow(s, X)^{\omega} \subseteq(t, Y)^{\omega} .
$$

If $\langle s, X\rangle$ is an $\mathbb{M}^{\star}$-condition, then we call $s$ the stem of the condition.

If $G$ is $\mathbb{M}^{\star}$-generic over $\mathbf{N}$, then $G$ induces in a canonical way an infinite partition $X_{G} \in(\omega)^{\omega}$ such that $\mathbf{N}[G]=\mathbf{N}\left[X_{G}\right]$, and therefore we consider

\footnotetext{
${ }^{8} \mathrm{~A}$ set $S$ has the Baire property if there is an Borel set $B$ such that the symmetric difference $S \triangle B$ is meager, where a meager set is the union of countably many nowhere dense sets.

${ }^{9}$ Cf. [El74] \& $[$ Ke95, §19.D].
} 
the partition $X_{G}$ as the generic object. We can reconstruct the original $G$ from $X_{G}$ by observing that

$$
\langle s, X\rangle \in G \Longleftrightarrow X_{G} \in(s, X)_{\mathbf{N}[G]}^{\omega} .
$$

Since the dual Ellentuck topology is innately connected with dual Mathias forcing, we choose the following notation for meager and comeager sets in the dual Ellentuck topology:

$$
\begin{aligned}
& A \in\left(\mathrm{m}_{0}^{\star}\right) \Longleftrightarrow A \text { is dual Ellentuck meager, and } \\
& A \in\left(\mathrm{m}_{1}^{\star}\right) \Longleftrightarrow A \text { is dual Ellentuck comeager, } \\
& \text { i.e., }(\omega)^{\omega} \backslash A \text { is dual Ellentuck meager. }
\end{aligned}
$$

Since $A$ is dual Ellentuck meager if and only if $A$ is completely dual Ramsey-null, $\left(\mathrm{m}_{0}^{\star}\right) \subseteq \mathcal{P}\left((\omega)^{\omega}\right)$ is also the ideal of completely dual Ramseynull sets.

The following fact gives two properties of dual Mathias forcing which also hold for Mathias forcing.

Fact 2.1. If $X_{G}$ is $\mathbb{M}^{\star}$-generic and $Y \in\left(X_{G}\right)^{\omega}$, then $Y$ is $\mathbb{M}^{\star}$-generic as well (we will call this property the homogeneity property); and therefore, dual Mathias forcing is proper. Moreover, for any sentence $\Phi$ of the forcing language $\mathbb{M}^{\star}$ and for any $\mathbb{M}^{\star}$-condition $\langle s, X\rangle$, there is an $\mathbb{M}^{\star}$-condition $\langle s, Y\rangle \leq\langle s, X\rangle$ such that $\langle s, Y\rangle \Vdash_{\mathbb{M} \star} \Phi$ or $\langle s, Y\rangle \vdash_{\mathbb{M} \star} \neg \Phi$ (this property is called pure decision).

Proof. For a proof, cf. [CaSi84, Theorem $5.5 \&$ Theorem 5.2].

As an immediate consequence we get that the set of dual Mathias generic partitions over every model $\mathbf{N}$ is either empty or a non-meager set which is completely dual Ramsey.

Like Mathias forcing, dual Mathias forcing has also a characteristic product form.

Let $\mathbb{U}^{\star}=\left\langle(\omega)^{\omega}, \leq\right\rangle$ be the partial order defined as follows:

$$
X \leq Y \Leftrightarrow X \sqsubseteq^{*} Y .
$$

$\mathbb{U}^{\star}$ is the natural dualization of $\mathcal{P}(\omega) /$ fin.

For a family $\mathfrak{E} \subseteq(\omega)^{\omega}$ we define the restricted dual Mathias forcing $\mathbb{M}_{\mathfrak{E}}^{\star}$ as follows. The conditions of $\mathbb{M}_{\mathfrak{E}}^{\star}=\left\langle M_{\mathfrak{E}}^{\star}, \leq\right\rangle$ are the $\mathbb{M}^{\star}$-conditions $\langle s, X\rangle$ such that $X \in \mathfrak{E}$.

Now we get

Fact 2.2. $\mathbb{M}^{\star}=\mathbb{U}^{\star} * \mathbb{M}_{G}^{\star}$, where $G$ is the canonical name for the $\mathbb{U}^{\star}$-generic object.

Proof. For a proof, cf. [Halb98-1, Fact 2.5]. 
2.4. Restricted dual Laver forcing. In order to define the forcing notion which will be investigated later on, we first have to give some notations.

For $T \subseteq(\mathbb{N})$ and $t \in T$ we define the successor set of $t$ in $T$ as follows:

$$
\operatorname{succ}_{T}(t):=\{u \in T: t \preccurlyeq u \wedge|u|=|t|+1\} .
$$

Let $\mathfrak{E} \subseteq(\omega)^{\omega}$ be any non-empty family (later on we investigate only the case when $\mathfrak{E}$ is an ultrafilter).

With respect to $\mathfrak{E}$, we define the dual Laver forcing restricted to $\mathfrak{E}$, denoted by $\mathbb{L}_{\mathfrak{E}}^{\star}=\left\langle L_{\mathfrak{E}}^{\star}, \leq\right\rangle$, as follows:

$(\alpha) p \in L_{\mathfrak{E}}^{\star}$ if and only if $p \subseteq(\mathbb{N})$ with the property that there is an $s \in p$ $($ denoted $\operatorname{stem}(p))$ such that for all $t \in p$ we have $s \preccurlyeq t$.

$(\beta)$ There exists a set $\left\{X_{t}^{p}: t \in p\right\} \subseteq \mathfrak{E}$ such that for $t \in p$ we have $t^{*} \sqsubseteq X_{t}^{p}$ and

$$
\operatorname{succ}_{p}(t)=\left\{u: u \in\left(t^{*}, X_{t}^{p}\right)^{(|t|+1) *}\right\} .
$$

Further, for $t, u \in p$ with $t \preccurlyeq u$ we have

$$
\left(u, X_{u}^{p}\right)^{\omega} \subseteq\left(t, X_{t}^{p}\right)^{\omega},
$$

and if $\operatorname{dom}(t)=\operatorname{dom}(u)$ and $t \sqsubseteq u$, then

$$
X_{t}^{p}=X_{u}^{p} \text {. }
$$

$(\gamma)$ For two $\mathbb{L}_{\mathfrak{E}}^{\star}$-conditions $p$ and $q$ we stipulate

$$
p \leq q \Longleftrightarrow p \subseteq q .
$$

Notice that $p \leq q$ implies $\operatorname{stem}(q) \preccurlyeq \operatorname{stem}(p)$ and hence, if $G \subseteq L_{\mathfrak{E}}^{\star}$ is $\mathbb{L}_{\mathfrak{E}^{-}}^{\star}$ generic over some $\mathbf{N}$, then the set $\{s: s=\operatorname{stem}(p)$ for some $p \in G\}$ forms in a canonical way a partition $X_{G} \in(\omega)^{\omega}$. Moreover, $\mathbf{N}[G]=\mathbf{N}\left[X_{G}\right]$ and therefore we may consider also the partition $X_{G}$ as the $\mathbb{L}_{\mathfrak{E}}^{\star}$-generic object.

For an $\mathbb{L}_{\mathfrak{E}}^{\star}$-condition $p$ we call a partition $X \in(\omega)^{\omega}$ a branch of $p$ if each $t \in(\mathbb{N})$ with $t^{*} \sqsubseteq X$ belongs to $p$.

Fact 2.3. If $X$ is a branch of the $\mathbb{L}_{\mathfrak{E}}^{\star}$-condition $p$ where $\operatorname{stem}(p)=s$ and $Y \in(s, X)^{\omega}$, then $Y$ is a branch of $p$, too.

Proof. This follows immediately from $(\beta)$.

2.5. Special ultrafilters on $(\omega)^{\omega}$. A family $\mathfrak{F}$ has the segment colouring property (or just scp) if for any $s \sqsubseteq X \in \mathfrak{F}$ with $|s|=n$ and for any colouring $\pi:(s, X)^{(n+k) *} \rightarrow r$, where $r$ and $n+k$ are positive natural numbers, there is a $Y \in(s, X)^{\omega} \cap \mathfrak{F}$ such that $(s, Y)^{(n+k) *}$ is monochromatic.

A family $\mathfrak{F} \subseteq(\omega)^{\omega}$ is an scp-filter if $\mathfrak{F}$ is a filter which has the segment colouring property.

In Section 7 we shall introduce the notion of game filters (from [Halb98-1]) and show that game filters are scp-filters.

Fact 2.4. If $\mathfrak{F} \subseteq(\omega)^{\omega}$ is an scp-filter, then $\mathfrak{F}$ is an ultrafilter. 
Proof. Let $\mathfrak{F} \subseteq(\omega)^{\omega}$ be an scp-filter and assume that there exists an $X \in$ $(\omega)^{\omega}$ such that for every $Y \in \mathfrak{F}, X \sqcap Y \in(\omega)^{\omega}$. Let $\pi:(\omega)^{n *} \rightarrow 2$ be such that $\pi(s)=0$ if $s \in(X)^{n *}$, otherwise $\pi(s)=1$. Because $\mathfrak{F}$ has the segment colouring property, we find a $Y \in \mathfrak{F}$ such that $\pi \uparrow(Y)^{n *}$ is constant. If $\pi \uparrow(Y)^{n *}=\{1\}$, then $X \sqcap Y \notin(\omega)^{\omega}$ which contradicts the assumption. Thus, $\pi \uparrow(Y)^{n *}=\{0\}$, which implies $X \in \mathfrak{F}$ and hence, the filter $\mathfrak{F}$ is maximal.

A family $\mathfrak{F}$ is diagonalizable if for any $\mathbb{L}_{\mathfrak{F}}^{\star}$-condition $p$, there is a partition $X \in \mathfrak{F}$ such that $X$ is a branch of $p$. Notice that a diagonalizable family can also be characterized by a two player game, where the $\mathbb{L}_{\mathfrak{F}}^{\star}$-condition $p$ can be considered as a strategy for player I.

A family $\mathfrak{F}$ is a Ramsey ${ }^{\star}$ filter if $\mathfrak{F}$ is a diagonalizable scp-filter.

In Footnote 5 we have defined Ramsey ultrafilters over $\omega$ in terms of colourings. This definition corresponds to the definition of scp-filters. On the other hand, Galvin and Shelah proved that Ramsey ultrafilters can be characterized as well by a two player game without a winning strategy for player I, where a winning strategy for player I is in fact a restricted Lavercondition (cf. [BaJu95, Theorem 4.5.3]). This definition of Ramsey ultrafilters corresponds to diagonalizable filters. It is possible that the notions of "scp-filters" and "diagonalizable filters" are equivalent, but this is still open.

Beyond the dualization of the notion of a Ramsey ultrafilter, the dualization process leading from $[\omega]^{\omega}$ to $(\omega)^{\omega}$ has interesting consequences for the spaces of ultrafilters on these spaces. These consequences belong to the asymmetrical aspects of the relationship between $[\omega]^{\omega}$ and $(\omega)^{\omega}$ and are the point of focus in [HalbLö $\infty]$.

2.6. Switching between reals and partitions. We fix $b:[\omega]^{2} \rightarrow \omega$ to be any arithmetic bijection between the set of pairs of natural numbers and $\omega$.

Let $x \in[\omega]^{\omega}$; then the set $\operatorname{trans}(x) \subseteq \omega$ is defined by

$$
\begin{aligned}
n \in \operatorname{trans}(x): \Longleftrightarrow \quad & \exists s \in \omega^{<\omega}(n=b(s(0), s(|s|-1)) \text { and } \\
& \forall k \in|s|-1(b(s(k), s(k+1)) \in x)) .
\end{aligned}
$$

As the name suggests, $\operatorname{trans}(x)$ is the set of codes of pairs in the transitive closure of the relation $b(k, \ell) \in x$. A real $x$ is called transitive if $\operatorname{trans}(x)=$ $x$.

Note that in general trans $(x) \subseteq x$ and that the relation

$$
R_{x}(k, \ell): \Longleftrightarrow b(k, \ell) \in \operatorname{trans}(x)
$$


is symmetric (by choice of the domain of $b$ ) and transitive. Thus, if $x \in[\omega]^{\omega}$, we can consider $x$ as a partition (by reflexivization of $R_{x}$ ) via

$$
n \sim_{x} m: \Longleftrightarrow n=m \text { or } b(n, m) \in \operatorname{trans}(x) .
$$

We call this partition the corresponding partition of $x \in[\omega]^{\omega}$, and denote it by $\operatorname{cp}(x)$. Note that $\operatorname{cp}(x) \in(\omega)^{\omega}$ if

$$
\forall k \exists n>k \forall m<n\left(\neg\left(n \sim_{x} m\right)\right)
$$

and further if $y \subseteq x$, then $\operatorname{cp}(y) \sqsupseteq \operatorname{cp}(x)$.

We encode a partition $X$ of $\omega$ by a real pc $(X)$ (the partition code of $X)$ as follows.

$$
\operatorname{pc}(X):=\left\{k \in \omega: \exists n \exists m\left(k=b(n, m) \wedge\left(n \sim_{X} m\right)\right)\right\} .
$$

Note that if $X \sqsubseteq Y$ then $\operatorname{pc}(X) \supseteq \operatorname{pc}(Y)$.

Notice that both the function pc and the function cp are arithmetic, and that they are in a sense inverse to each other:

Observation 2.5. For every $X \in(\omega)^{\omega}$ and every $x \in[\omega]^{\omega}$ the following hold:

(i) $\operatorname{cp}(\operatorname{pc}(X))=X$, and

(ii) if $x$ is transitive, then $\operatorname{pc}(\operatorname{cp}(x))=x$.

Now, a set $A \subseteq[\omega]^{\omega}$ has the dual Ramsey property (or just is dual Ramsey) if and only if the set $\left\{X \in(\omega)^{\omega}: \exists x \in A(X=\operatorname{cp}(x))\right\}$ has the dual Ramsey property. By Observation 2.5, this is equivalent to saying that the set $\left\{X \in(\omega)^{\omega}: \operatorname{pc}(X) \in A\right\}$ has the dual Ramsey property.

By the definition of the dual Ramsey property we have that every $\boldsymbol{\Sigma}_{n}^{1}$ set is dual Ramsey if and only if every $\boldsymbol{\Pi}_{n}^{1}$ set is dual Ramsey. Furthermore, we have by [Halb98-1, Lemma 7.2] that if every $\boldsymbol{\Sigma}_{n}^{1}$ set is dual Ramsey then every $\boldsymbol{\Sigma}_{n}^{1}$ set has the classical Ramsey property.

As a matter of fact we like to mention the following

Proposition 2.6. If every $\boldsymbol{\Delta}_{n}^{1}$ set has the dual Ramsey property, then every $\boldsymbol{\Delta}_{n}^{1}$ set has the Ramsey property.

Proof. Suppose $A$ is a $\boldsymbol{\Delta}_{n}^{1}$ set of reals. Let $\varphi$ be a $\boldsymbol{\Sigma}_{n}^{1}$ formula and $\psi$ be a $\boldsymbol{\Pi}_{n}^{1}$ formula witnessing this, i.e.,

$$
x \in A \Longleftrightarrow \varphi(x) \Longleftrightarrow \psi(x) .
$$

To show that $A$ is Ramsey we define a different $\boldsymbol{\Delta}_{n}^{1}$ set by formulae $\varphi^{*}$ and $\psi^{*}$ as follows:

$$
\begin{aligned}
\varphi^{*}(v) & : \Longleftrightarrow \exists w(w=\operatorname{Min}(\operatorname{cp}(v)) \wedge \varphi(w)), \\
\psi^{*}(v) & \Longleftrightarrow \Longleftrightarrow \forall w(w=\operatorname{Min}(\operatorname{cp}(v)) \rightarrow \psi(w)) .
\end{aligned}
$$


Obviously, $\varphi^{*}$ is $\boldsymbol{\Sigma}_{n}^{1}$ and $\psi^{*}$ is $\boldsymbol{\Pi}_{n}^{1}$, and since $\operatorname{Min}(\operatorname{cp}(v))$ is uniquely determined for each $v$, these two formulae are equivalent and hence define a $\boldsymbol{\Delta}_{n}^{1}$ set $A^{*}$ of reals. The rest of the proof is exactly as in [Halb98-1, Lemma 7.2].

And as a corollary we get

Corollary 2.7. If every $\boldsymbol{\Delta}_{2}^{1}$ set is dual Ramsey, then every $\boldsymbol{\Sigma}_{2}^{1}$ set is Ramsey.

Proof. This follows immediately from Proposition 2.6 by Theorem 1.2.

2.7. Descriptive Set Theory of the Cabal. For our results in Section 6 we shall need some basic notions of the Descriptive Set Theory of the Cabal Seminar. Everything we lay out here can be found in [Mo80], our account is just for the convenience of the more combinatorially oriented reader who might be unfamiliar with the language of the Cabal.

We shall presuppose basic knowledge with the standard notation for determinacy and the elementary results of the theory of perfect information games as outlined in [Kan94, §27].

Let $X$ be a set of reals and $\alpha \in$ Ord. Any surjective function $\varphi: X \rightarrow \alpha$ is called a norm on $X$. The ordinal $\alpha$ is called the length of $\varphi$. A family $\Phi:=\left\langle\varphi_{n}: n \in \omega\right\rangle$ of norms on $X$ is called a scale on $X$ if for every sequence $\left\langle x_{i}: i \in \omega\right\rangle \subseteq X$ and every $n \in \omega$ the following holds: If $\left\langle\varphi_{n}\left(x_{i}\right): i \in \omega\right\rangle$ is eventually constant, say, equal to $\lambda_{n}$, then $x:=\lim _{i \in \omega} x_{i} \in X$ and $\varphi_{n}(x) \leq$ $\lambda_{n} \cdot{ }^{10}$

Let $\Gamma$ be any pointclass, $\varphi$ any norm on $X$, and $\Phi$ any scale on $X$. We shall call $\varphi$ a $\Gamma$ norm if there are two relations $R$ and $R^{*}$ in $\Gamma$ such that:

$$
y \in X \Rightarrow \forall x\left((x \in X \wedge \varphi(x) \leq \varphi(y)) \Longleftrightarrow R(x, y) \Longleftrightarrow \neg R^{*}(x, y)\right) \text {. }
$$

We call a scale $\Phi$ a $\Gamma$ scale if all norms $\varphi_{n}$ occurring in $\Phi$ are $\Gamma$ norms, uniformly in $n .{ }^{11}$ We shall say that a set $X$ admits a $\Gamma$ norm (a $\Gamma$ scale) if there is a norm (a scale) on $X$ that is a $\Gamma$ norm (a $\Gamma$ scale).

The fundamental theorems connecting determinacy, norms and scales are the "Periodicity Theorems" of [AdMo68], [Mar68] and [Mo71]. In the following we shall need the first two Periodicity Theorems in special cases:

First Periodicity Theorem 2.8. Suppose that $\operatorname{Det}\left(\boldsymbol{\Delta}_{2 n}^{1}\right)$ holds and $x \in$ $[\omega]^{\omega}$ is a real. Then every $\Pi_{2 n+1}^{1}(x)$ set admits a $\Pi_{2 n+1}^{1}(x)$ norm.

Second Periodicity Theorem 2.9. Suppose that $\operatorname{Det}\left(\boldsymbol{\Delta}_{2 n}^{1}\right)$ holds and $x \in$ $[\omega]^{\omega}$ is a real. Then every $\Pi_{2 n+1}^{1}(x)$ set admits a $\Pi_{2 n+1}^{1}(x)$ scale.

\footnotetext{
${ }^{10}$ For the basic theory of scales, cf. [KeMo78].

${ }^{11} \mathrm{~A}$ more precise definition can be found in [Mo80, p. 228].
} 
For proofs of these theorems (in a much more general formulation), we refer the reader to $[\mathbf{M o 8 0}, 6 \mathrm{~B} .1 \&$ \& C.3].

We define (for every $n \in \omega$ ) the projective ordinals by

$$
\delta_{n}^{1}:=\sup \left\{\|\leq\|: \leq \text { is a } \Delta_{n}^{1} \text { prewellordering on }[\omega]^{\omega}\right\},
$$

and note that for every $\Pi_{2 n+1}^{1}$ complete set the length of every $\Pi_{2 n+1}^{1}$ norm on it is exactly $\boldsymbol{\delta}_{2 n+1}^{1}([\mathbf{M o 8 0}, 4$ C.14] $)$.

Let $P_{2 n+1}^{x}$ be a $\Pi_{2 n+1}^{1}(x)$ complete set of reals. Assuming $\operatorname{Det}\left(\boldsymbol{\Delta}_{2 n}^{1}\right)$ we get a $\Pi_{2 n+1}^{1}(x)$ scale $\Phi^{x}=\left\{\varphi_{m}^{x}: m \in \omega\right\}$ for $P_{2 n+1}^{x}$ by Theorem 2.9.

For any real $y \in P_{2 n+1}^{x}$, we denote by $\Phi^{x}(y)$ the sequence of ordinals determined by the scale, i.e., $\Phi^{x}(y)=\left\langle\varphi_{m}^{x}(y): m \in \omega\right\rangle$.

Now let

$$
T_{2 n+1}^{x}:=\left\{\left\langley\left\lceil m, \Phi^{x}(y)\lceil m\rangle: y \in P_{2 n+1}^{x}, m \in \omega\right\}\right.\right.
$$

be the tree associated to $\Phi^{x}$. By the remark about the lengths of norms, it is a tree on $\omega \times \delta_{2 n+1}^{1}$. If $x$ is any recursive real, we write $T_{2 n+1}$ instead of $T_{2 n+1}^{x}$.

The model $\mathbf{L}\left[T_{2 n+1}\right]$ can be seen as an analogue of the constructible universe $\mathbf{L}$ in the odd projective levels: The (Shoenfield) $\Pi_{1}^{1}$ scale for a $\Pi_{1}^{1}$ complete set is in $\mathbf{L}$, hence $\mathbf{L}\left[T_{1}\right]=\mathbf{L} .{ }^{12}$ Indeed, the reals of $\mathbf{L}\left[T_{2 n+1}\right]$ are exactly the reals of $\mathbf{M}_{2 n}$, the canonical iterable inner model with $2 n$ Woodin cardinals. ${ }^{13}$

Moreover, not just the reals of the models, but the models $\mathbf{L}\left[T_{2 n+1}\right]$ themselves are independent of the choices of the particular $\Pi_{2 n+1}^{1}$ complete set and the scale on it, as has been shown by Becker and Kechris in [BeKe84, Theorem 1 \& 2]:

Theorem 2.10. Assume PD and let $x \in[\omega]^{\omega}$ be a real. If $P$ and $Q$ are $\Pi_{2 n+1}^{1}(x)$ complete sets, $\Phi$ and $\Psi$ are scales on $P$ and $Q$, respectively, and $T$ and $S$ are the trees associated to $\Phi$ and $\Psi$, respectively. Then $\mathbf{L}[T]=\mathbf{L}[S]$.

Another consequence of determinacy which will be mentioned only briefly to simplify notation is the existence of largest countable sets of certain (lightface) complexity classes:

Theorem 2.11. Let $x \in[\omega]^{\omega}$ be a real. Suppose that $\operatorname{Det}\left(\Delta_{2 n}^{1}(x)\right)$ holds. Then there is a largest countable $\Sigma_{2 n+2}^{1}(x)$ set which will be denoted by $C_{2 n+2}(x){ }^{14}$

Proof. Cf. [KeMo72, Theorem 2].

\footnotetext{
${ }^{12}$ For a proof, cf. [KeMo78, 9C].

${ }^{13}$ Combine [St95, Corollary 4.9] with [HarKe81, Theorem 7.2.1].

${ }^{14}$ As for the trees $T_{2 n+1}$, we shall omit the parameter $x$ and write $C_{2 n+1}$ if the real $x$ is recursive.
} 


\section{On Ramsey ${ }^{\star}$ ultrafilters.}

In this section we show that Ramsey ${ }^{\star}$ ultrafilters exist if we assume $\mathrm{CH}$ and that in general both existence and non-existence of Ramsey ${ }^{\star}$ ultrafilters are consistent with ZFC.

First we show that an scp-ultrafilter induces in a canonical way a Ramsey filter on $\omega$.

Fact 3.1. If $\mathfrak{F} \subseteq(\omega)^{\omega}$ is an scp-ultrafilter, then $\{\operatorname{Min}(X): X \in \mathfrak{F}\} \backslash\{0\}$ is a Ramsey filter on $\omega$.

Proof. For positive natural numbers $n$ and $r$ let $\tau:[\omega]^{n} \rightarrow r$ be any colouring. We define $\pi:(\omega)^{n *} \rightarrow r$ by stipulating $\pi(s):=\tau\left(\operatorname{Min}\left(s^{*}\right) \backslash\{0\}\right)$. It is easy to see that if $\pi\left\lceil(X)^{n *}\right.$ is constant for an $X \in \mathfrak{F}$, then $\tau \uparrow[\operatorname{Min}(X) \backslash\{0\}]^{n}$ is constant, too.

Proposition 3.2. It is consistent with ZFC that there are no scp-ultrafilters.

Proof. Kunen proved (cf. [Je78, Theorem 91]) that it is consistent with ZFC that there are no Ramsey filters on $\omega$. Therefore, by Fact 3.1, in a model of ZFC in which there are no Ramsey filters, there are also no scpultrafilters.

Let $\mathbb{U}^{\star}=\left\langle(\omega)^{\omega}, \leq\right\rangle$ be the partial order defined as in Subsection 2.3. It is easy to see that the forcing notion $\mathbb{U}^{\star}$ is $\sigma$-closed (this is part of Fact 2.3 of [Halb98-1]).

Lemma 3.3. If $G$ is $\mathbb{U}^{\star}$-generic over $\mathbf{V}$, then $G$ is an scp-ultrafilter in $\mathbf{V}[G]$.

Proof. Let $s \in(\mathbb{N})$ and $k \in \omega$ with $|s|=n$ and $n+k>0$. Further, let $\pi:(\omega)^{(n+k) *} \rightarrow r$ be any colouring and for $s \sqsubseteq X \in(\omega)^{\omega}$ let

$$
H_{\pi(s, X)}:=\left\{Y \in(s, X)^{\omega}: \pi(s, X)\left\lceil(s, Y)^{(n+k) *} \text { is constant }\right\} .\right.
$$

By the main result of $[\mathbf{H a l b} \infty]$ and its proof, for every dual Ellentuck neighbourhood $(s, X)^{\omega}$ and for any colouring $\pi:(s, X)^{(n+k) *} \rightarrow r$, there is a $Y \in(s, X)^{\omega}$ such that $\pi \uparrow(s, Y)^{(n+k) *}$ is constant. Hence, for any dual Ellentuck neighbourhood $(s, X)^{\omega}$ and for any colouring $\pi:(\omega)^{(n+k) *} \rightarrow r$, the set $H_{\pi(s, X)}$ is dense below $X$. Because every such colouring $\pi$ can be encoded by a real and $\mathbb{U}^{\star}$ is $\sigma$-closed, the forcing notion $\mathbb{U}^{\star}$ does not add any colouring $\pi$, which implies, because $G$ meets each dense set, that $G$ is an scp-ultrafilter in $\mathbf{V}[G]$.

We can prove with similar arguments:

Lemma 3.4. If $G$ is $\mathbb{U}^{\star}$-generic over $\mathbf{V}$, then $G$ is a diagonalizable ultrafilter in $\mathbf{V}[G]$. 
Proof. Let $\dot{p}$ be a $\mathbb{U}^{\star}$-name such that

$$
\Vdash_{\mathbb{U} \star} \text { " } \dot{p} \text { is an } \mathbb{L}_{\boldsymbol{G}}^{\star} \text {-condition", }
$$

where $\boldsymbol{G}$ is the canonical name for the $\mathbb{U}^{\star}$-generic object, and let $X$ be any $\mathbb{U}^{\star}$-condition. Because $\dot{p}$ can be encoded by a real number and $\mathbb{U}^{\star}$ is $\sigma$-closed, there is a $\mathbb{U}^{\star}$-condition $Y \leq X$ and a real $p^{\prime} \in \mathbf{V}$ such that $Y \Vdash_{\mathbb{U} \star} p^{\prime}=\dot{p}$, which implies $Y \sqsubseteq^{*} \operatorname{succ}_{p^{\prime}}(t)$ for every $t \in p^{\prime}$. By induction one can construct a $Z \sqsubseteq^{*} Y$ such that $Z$ is a branch of $p^{\prime}$ and therefore,

$$
Z \vdash_{\mathbb{U} \star} \text { "there is a branch of } \dot{p} \text { which belongs to } G \text { ". }
$$

Since $Z \leq X$, this completes the proof.

Proposition 3.5. Assume $\mathrm{CH}$, then there is a Ramsey ${ }^{\star}$ ultrafilter.

Proof. Assume $\mathbf{V}=\mathrm{CH}$. Let $\chi$ be large enough such that $\mathcal{P}\left((\omega)^{\omega}\right) \in H(\chi)$, i.e., the power set of $(\omega)^{\omega}$ (in $\mathbf{V}$ ) is hereditarily of size $<\chi$. Let $\mathbf{N}$ be an elementary submodel of $\langle H(\chi), \in\rangle$ containing all reals of $\mathbf{V}$ with $|\mathbf{N}|=2^{\aleph_{0}}$. We consider the forcing notion $\mathbb{U}^{\star}$ in the model $\mathbf{N}$. Because $|\mathbf{N}|=2^{\aleph_{0}}$, in $\mathbf{V}$ there is an enumeration $\left\{D_{\alpha} \subseteq(\omega)^{\omega}: \alpha<2^{\aleph_{0}}\right\}$ of all dense sets of $\mathbb{U}^{\star}$ which lie in $\mathbf{N}$. Since $\mathbb{U}^{\star}$ is $\sigma$-closed and because $\mathbf{V} \models \mathbf{C H}, \mathbb{U}^{\star}$ is $2^{\aleph_{0}}$-closed in $\mathbf{V}$ and therefore we can construct a descending sequence $\left\{p_{\alpha}: \alpha<2^{\aleph_{0}}\right\}$ in $\mathbf{V}$ such that $p_{\alpha} \in D_{\alpha}$ for each $\alpha<2^{\aleph_{0}}$. Let $G:=\left\{p \in(\omega)^{\omega}: p_{\alpha} \sqsubseteq\right.$ $p$ for some $\left.p_{\alpha}\right\}$, then $G$ is $\mathbb{U}^{\star}$-generic over $\mathbf{N}$. By Lemma 3.3 and Lemma 3.4 we have $\mathbf{N}[G] \models$ "there is a Ramsey ${ }^{\star}$ ultrafilter", and because $\mathbf{N}$ contains all reals of $\mathbf{V}$ and every function $f:(\omega)^{n *} \rightarrow r$ (where $n, r \in \omega$ ) and every $\mathbb{L}_{G}^{\star}$-condition $p$ can be encoded by a real number, the Ramsey ${ }^{\star}$ ultrafilter in $\mathbf{N}[G]$ is also a Ramsey ${ }^{\star}$ ultrafilter in $\mathbf{V}$, which completes the proof.

\section{On $\mathbb{L}_{\mathfrak{F}}^{\star}$ and $\mathbb{M}_{\mathfrak{F}}^{\star}$ for Ramsey filters $\mathfrak{F}$.}

In this section, $\mathfrak{F} \subseteq(\omega)^{\omega}$ denotes always a Ramsey ${ }^{\star}$ ultrafilter.

We shall show that the forcing notions $\mathbb{L}_{\mathfrak{F}}^{\star}$ and $\mathbb{M}_{\mathfrak{F}}^{\star}$ are equivalent and that both forcing notions have pure decision and the homogeneity property (this means that coarsenings of generic objects remain generic, see Fact 2.1). We show first that $\mathbb{M}_{\mathfrak{F}}^{\star}$ has pure decision and the homogeneity property. To show this we will follow [Halb98-1, Section 4].

If $s \in(\mathbb{N})$ and $s \sqsubseteq X \in \mathfrak{F}$, then we call the dual Ellentuck neighbourhood $(s, X)^{\omega}$ an $\mathfrak{F}$-dual Ellentuck neighbourhood and write $(s, X)_{\mathfrak{F}}^{\omega}$ to emphasize that $X \in \mathfrak{F}$. A set $\mathcal{O} \subseteq(\omega)^{\omega}$ is called $\mathfrak{F}$-open if $\mathcal{O}$ can be written as the union of some $\mathfrak{F}$-dual Ellentuck neighbourhoods.

For $s \in(\mathbb{N})$ remember that $s^{*}=s \cup\{\{\operatorname{dom}(s)\}\}$.

Let $\mathcal{O} \subseteq(\omega)^{\omega}$ be an $\mathfrak{F}$-open set. Call $(s, X)_{\mathfrak{F}}^{\omega}$ good (with respect to $\mathcal{O}$ ), if for some $Y \in(s, X)_{\mathfrak{F}}^{\omega} \cap \mathfrak{F},(s, Y)_{\mathfrak{F}}^{\omega} \subseteq \mathcal{O}$; otherwise call it bad. Note that if $(s, X)_{\mathfrak{F}}^{\omega}$ is bad and $Y \in(s, X)_{\mathfrak{F}}^{\omega} \cap \mathfrak{F}$, then $(s, Y)_{\mathfrak{F}}^{\omega}$ is bad, too. We call $(s, X)_{\mathfrak{F}}^{\omega}$ 
ugly if $\left(t^{*}, X\right)_{\mathfrak{F}}^{\omega}$ is bad for all $s \preccurlyeq t^{*} \sqsubseteq X$ with $|t|=|s|$. Note that if $(s, X)_{\mathfrak{F}}^{\omega}$ is ugly, then $(s, X)_{\mathfrak{F}}^{\omega}$ is bad.

Lemma 4.1. Let $\mathfrak{F} \subseteq(\omega)^{\omega}$ be a Ramsey $y^{\star}$ ultrafilter and $\mathcal{O} \subseteq(\omega)^{\omega}$ an $\mathfrak{F}$ open set. If $(s, X)_{\mathfrak{F}}^{\omega}$ is bad (with respect to $\mathcal{O}$ ), then there is a $Z \in(s, X)_{\mathfrak{F}}^{\omega}$ such that $(s, Z)_{\mathfrak{F}}^{\omega}$ is ugly.

Proof. We begin by constructing an $\mathbb{L}_{\mathfrak{F}}^{\star}$-condition $p$. Let $s_{0}$ be such that $s \preccurlyeq s_{0}^{*} \sqsubseteq X$ and $|s|=\left|s_{0}\right|$, and put $\operatorname{stem}(p):=s_{0}$. If there is an $Y \in$ $\left(s_{0}^{*}, X\right)_{\mathfrak{F}}^{\omega} \cap \mathfrak{F}$ such that $\left(s_{0}^{*}, Y\right)_{\mathfrak{F}}^{\omega} \subseteq \mathcal{O}$, then $X_{s_{0}}:=Y$, otherwise, $X_{s_{0}}:=X$. Let $s_{n+1}^{*} \preccurlyeq\left(s_{n} \sqcap X_{s_{n}}\right)$ be such that $\left|s_{n+1}\right|=\left|s_{n}\right|+1=|s|+n+1$ and let $\left\{t_{i}: i \leq h\right\}$ be an enumeration of all $t$ such that $s_{0} \preccurlyeq t \sqsubseteq s_{n+1},|t|=|s|$ and $\operatorname{dom}(t)=\operatorname{dom}\left(s_{n+1}\right)$. Further let $Y^{-1}:=X_{s_{n}}$. Now choose for each $i \leq h$ a partition $Y^{i} \in \mathfrak{F}$ such that $Y^{i} \sqsubseteq Y^{i-1}, s_{n+1}^{*} \preccurlyeq Y^{i}$ and $\left(\left(t_{i}\right)^{*}, Y^{i}\right)_{\mathfrak{F}}^{\omega}$ is bad or $\left(\left(t_{n}^{i}\right)^{*}, Y^{i}\right)_{\mathfrak{F}}^{\omega} \subseteq \mathcal{O}$ and finally, let $X_{s_{n+1}}:=Y^{h}$.

Put $p:=\left\{t \in(\mathbb{N}): s_{0} \preccurlyeq t^{*} \sqsubseteq s_{n}^{*}\right.$ for some $\left.n \in \omega\right\}$. Since $\mathfrak{F}$ is diagonalizable, there is a partition $Y \in \mathfrak{F}$ which is a branch of $p$. We may assume that $s_{0} \preccurlyeq Y$. Define $S_{Y}:=\left\{t: s \preccurlyeq t^{*} \sqsubseteq Y \wedge|t|=|s|\right\}$; then, by the construction of $p$, for all $t \in S_{Y}$ we have either $\left(t^{*}, Y\right)_{\mathfrak{F}}^{\omega}$ is bad or $\left(t^{*}, Y\right)_{\mathfrak{F}}^{\omega} \subseteq \mathcal{O}$. Now let $B_{0}:=\left\{t \in S_{Y}:(t, Y)_{\mathfrak{F}}^{\omega}\right.$ is bad $\}$ and $B_{1}:=\left\{t \in S_{Y}:\left(t^{*}, Y\right)_{\mathfrak{F}}^{\omega} \subseteq \mathcal{O}\right\}=S_{Y} \backslash B_{0}$. Because $\mathfrak{F}$ is an scp-filter, there is a partition $Z \in(s, Y)_{\mathfrak{F}}^{\omega} \cap \mathfrak{F}$ such that $S_{Z} \subseteq B_{0}$ or $S_{Z} \subseteq B_{1}$. If we are in the latter case, we have $(s, Z)_{\mathfrak{F}}^{\omega} \subseteq \mathcal{O}$, which is a contradiction to our assumption that $(s, X)_{\mathfrak{F}}^{\omega}$ is bad. So, we must have $S_{Z} \subseteq B_{0}$, which implies that $(s, Z)_{\mathfrak{F}}^{\omega}$ is ugly and completes the proof of the Lemma.

Lemma 4.2. If $\mathfrak{F}$ is a Ramsey ${ }^{\star}$ ultrafilter and $\mathcal{O} \subseteq(\omega)^{\omega}$ is an $\mathfrak{F}$-open set, then for every $\mathfrak{F}$-dual Ellentuck neighbourhood $(s, X)_{\mathfrak{F}}^{\omega}$, there is a $Y \in$ $(s, X)_{\mathfrak{F}}^{\omega} \cap \mathfrak{F}$ such that $(s, Y)_{\mathfrak{F}}^{\omega} \subseteq \mathcal{O}$ or $(s, Y)_{\mathfrak{F}}^{\omega} \cap \mathcal{O} \cap \mathfrak{F}=\emptyset$.

Proof. If $(s, X)_{\mathfrak{F}}^{\omega}$ is good, then we are done. Otherwise, we can construct an $\mathbb{L}_{\mathfrak{F}}^{\star}$-condition $p$ in a similar way as in Lemma 4.1 , such that for any branch $Y$ of $p$ which belongs to $\mathfrak{F}$ we have the following: For each $t$ with $s \preccurlyeq t^{*} \sqsubseteq Y$, the set $\left(t^{*}, Y\right)_{\mathfrak{F}}^{\omega}$ is bad. We claim that $(s, Y)_{\mathfrak{F}}^{\omega} \cap \mathcal{O} \cap \mathfrak{F}=\emptyset$. Take any $Z \in(s, Y)_{\mathfrak{F}}^{\omega} \cap \mathcal{O} \cap \mathfrak{F}$. Because $\mathcal{O}$ is $\mathfrak{F}$-open we find a $t \preccurlyeq Z$ such that $\left(t^{*}, Z\right)_{\mathfrak{F}}^{\omega} \subseteq \mathcal{O}$. Because $s \preccurlyeq t^{*} \sqsubseteq Y$ we have by construction that $\left(t^{*}, Y\right)_{\mathfrak{F}}^{\omega}$ is bad. Hence, there is no $Z \in\left(t^{*}, Y\right)_{\mathfrak{F}}^{\omega}$ such that $\left(t^{*}, Z\right)_{\mathfrak{F}}^{\omega} \subseteq \mathcal{O}$. This completes the proof.

Now we can show that $\mathbb{M}_{\mathfrak{F}}^{\star}$ has pure decision and the homogeneity property.

Theorem 4.3. Let $\mathfrak{F}$ be a Ramsey ultrafilter and let $\Phi$ be a sentence of the forcing language. For any $\mathbb{M}_{\mathfrak{F}}^{\star}$-condition $\langle s, X\rangle$ there is a $\mathbb{M}_{\mathfrak{F}}^{\star}$-condition $\langle s, Y\rangle \leq\langle s, X\rangle$ such that $\langle s, Y\rangle \Vdash_{\mathbb{M}_{\mathfrak{F}}^{\star}} \Phi$ or $\langle s, Y\rangle \Vdash_{\mathbb{M}_{\mathfrak{F}}^{\star}} \neg \Phi$. 
Proof. The proof is same as the proof of [Halb98-1, Theorem 4.3], using Lemma 4.2.

The next theorem shows in fact that if $\mathfrak{F}$ is a Ramsey ${ }^{\star}$ ultrafilter, then $\mathbb{M}_{\mathfrak{F}}^{\star}$ is proper.

Theorem 4.4. Let $\mathfrak{F} \subseteq(\omega)^{\omega}$ be a Ramsey ${ }^{\star}$ ultrafilter, then $\mathbb{M}_{\mathfrak{F}}^{\star}$ has the homogeneity property.

Proof. The proof is same as the proof of [Halb98-1, Theorem 4.4], using Lemma 4.2.

In order to show that $\mathbb{M}_{\mathfrak{F}}^{\star}$ and $\mathbb{L}_{\mathfrak{F}}^{\star}$ are equivalent if $\mathfrak{F}$ is a Ramsey ${ }^{\star}$ ultrafilter, we define first some special $\mathbb{L}_{\mathfrak{F}}^{\star}$-conditions.

An $\mathbb{L}_{\mathfrak{F}^{\star}}^{\star}$-condition $p$ is called uniform if there is a partition $X \in \mathfrak{F}$ such that $(t, X)^{\omega}=\left(t, X_{t}^{p}\right)^{\omega}$ for every $t \in p$; this partition is denoted by $\mathrm{u}(p)$. These conditions roughly correspond to the simple conditions of $[\mathbf{J u S h} 89$, Definition 1.10].

Lemma 4.5. If $\mathfrak{F}$ is a Ramsey $y^{\star}$ ultrafilter, then the set of all uniform $\mathbb{L}_{\mathfrak{F}}^{\star}$ conditions is dense and open in $L_{\mathfrak{F}}^{\star}$.

Proof. Let $p \in L_{\mathfrak{F}}^{\star}$ with $s=\operatorname{stem}(p)$, then, since $\mathfrak{F}$ is diagonalizable, there is an $X \in \mathfrak{F}$ which is a branch of $p$. Let $q$ be the uniform condition with $\mathrm{u}(q)=X$ and $\operatorname{stem}(q)=s$. Note that $X$ is a branch of $q$. By Fact 2.3, each $Y \in(s, X)^{\omega}$ is also a branch of $p$, which implies that $q \leq p$.

Theorem 4.6. If $\mathfrak{F}$ is a Ramsey $y^{\star}$ ultrafilter, then $\mathbb{M}_{\mathfrak{F}}^{\star}$ and $\mathbb{L}_{\mathfrak{F}}^{\star}$ are forcing equivalent.

Proof. Let $I:=\left\{p \in L_{\mathfrak{F}}^{\star}: p\right.$ is uniform $\}$ and define

$$
\begin{array}{cccc}
j: I & \longrightarrow & M_{\mathfrak{F}}^{\star} \\
p & \longmapsto & \langle\operatorname{stem}(p), \mathrm{u}(p)\rangle,
\end{array}
$$

then it is easily checked that $j$ is a dense embedding and because (by Lemma 4.5) $I$ is dense open in $L_{\mathfrak{F}}^{\star}$, this completes the proof.

This is the promised dualization of Theorem 1.5 and possibly one step towards a proof of Conjecture 1.4.

\section{The dual Ramsey property for simple pointclasses.}

In the following we will show that it is consistent with ZFC that the sets in the first levels of the projective hierarchy are dual Ramsey. We begin with the analytic sets:

Because $\mathbb{M}^{\star}$ has pure decision and the homogeneity property, one can show the pretty straightforward 
Fact 5.1. Analytic sets are completely dual Ramsey.

Proof. Let $A$ be an arbitrary $\Sigma_{1}^{1}(a)$ set with parameter $a \in[\omega]^{\omega}$ and let $(s, Y)^{\omega}$ be any dual Ellentuck neighbourhood and $\langle s, Y\rangle$ the corresponding $\mathbb{M}^{\star}$-condition. Take a countable model $\mathbf{N}$ of a sufficiently large fragment of ZFC which contains $Y$ and $a$. Let $\boldsymbol{X}_{G}$ be the canonical name for the $\mathbb{M}^{\star}$-generic object. Because $\mathbb{M}^{\star}$ has pure decision we find an $\mathbb{M}^{\star}$-condition $\langle s, Z\rangle \leq\langle s, Y\rangle$ which decides " $\boldsymbol{X}_{G} \in \dot{A}$ ". Since $\mathbf{N}$ is countable, there is an $X \in(s, Z)^{\omega}$ which is $\mathbb{M}^{\star}$-generic over $\mathbf{N}$ and because every $X^{\prime} \in(s, X)^{\omega}$ is also $\mathbb{M}^{\star}$-generic we have

$$
\mathbf{N}[X] \mid="(s, X)^{\omega} \subseteq A \text { or }(s, X)^{\omega} \cap A=\emptyset " .
$$

Because $A$ and $(s, Y)^{\omega}$ were arbitrary and $\Sigma_{1}^{1}$ sets are absolute between $\mathbf{V}$ and $\mathbf{N}$, we are done.

Note that Fact 5.1 is verified without any reference to forcing by looking at the unfolded version of the Banach-Mazur game for the dual Ellentuck topology. ${ }^{15}$

Remember that according to Proposition 2.6 if every $\boldsymbol{\Delta}_{2}^{1}$ set is dual Ramsey then every $\boldsymbol{\Delta}_{2}^{1}$ set has the classical Ramsey property. Because it is not provable in ZFC that every $\boldsymbol{\Delta}_{2}^{1}$ set is Ramsey, it is also not provable in ZFC that every $\boldsymbol{\Delta}_{2}^{1}$ set is dual Ramsey (e.g., $\mathbf{L} \models$ "There is a $\boldsymbol{\Delta}_{2}^{1}$ set which is not dual Ramsey"). On the other hand we have

Fact 5.2. The following theories are equiconsistent:

(a) ZFC.

(b) $\mathrm{ZFC}+\mathrm{CH}+$ every $\boldsymbol{\Sigma}_{2}^{1}$ set is dual Ramsey.

(c) ZFC $+2^{\aleph_{0}}=\aleph_{2}+$ every $\boldsymbol{\Sigma}_{2}^{1}$ set is dual Ramsey.

Proof. We get both (b) and (c) by an iteration (of length $\omega_{1}$ and $\omega_{2}$ respectively) of dual Mathias forcing with countable support, starting from $\mathbf{L}$ (cf. also [Halb98-1, Theorems $6.2 \& 6.3])$.

Remember that $\left(\mathrm{m}_{0}^{\star}\right)=\left\{A \subseteq(\omega)^{\omega}: A\right.$ is completely dual Ramsey-null $\}$ and let

$$
\operatorname{add}\left(\mathrm{m}_{0}^{\star}\right):=\min \left\{|\mathfrak{E}|: \mathfrak{E} \subseteq\left(\mathrm{m}_{0}^{\star}\right) \wedge \bigcup \mathfrak{E} \notin\left(\mathrm{m}_{0}^{\star}\right)\right\}
$$

and

$$
\operatorname{cov}\left(\mathrm{m}_{0}^{\star}\right):=\min \left\{|\mathfrak{E}|: \mathfrak{E} \subseteq\left(\mathrm{m}_{0}^{\star}\right) \wedge \bigcup \mathfrak{E}=(\omega)^{\omega}\right\} .
$$

In [Halb98-2] it is shown that $\operatorname{add}\left(\mathrm{m}_{0}^{\star}\right)=\operatorname{cov}\left(\mathrm{m}_{0}^{\star}\right)=\mathfrak{H}$, where $\mathfrak{H}$ is the dual shattering cardinal. If $\left(\mathrm{m}_{0}\right)$ denotes the ideal of classical completely Ramsey-null sets, then we get the analogous result, namely $\operatorname{add}\left(\mathrm{m}_{0}\right)=$ $\operatorname{cov}\left(m_{0}\right)=\mathfrak{h}$, where $\mathfrak{h}$ is the shattering cardinal (cf. $\left.[\mathbf{P l} 186]\right)$. Because every

${ }^{15}$ Cf. [Ke95, Theorem (21.8)]. 
$\boldsymbol{\Sigma}_{2}^{1}$ set is the union of $\aleph_{1}$ Borel sets (cf. [Je78, Theorem 95]), it is easy to see that $\mathfrak{H}>\aleph_{1}$ implies that every $\boldsymbol{\Sigma}_{2}^{1}$ set is even completely dual Ramsey (and the analogous result holds for the classical Ramsey property with respect to $\mathfrak{h})$. Now, an $\omega_{2}$-iteration with countable support of dual Mathias forcing starting from $\mathbf{L}$ yields a model in which $\mathfrak{H}=\aleph_{2}$ (cf. [Halb98-2]). Thus, this provides another proof that "Every $\boldsymbol{\Sigma}_{2}^{1}$ set is dual Ramsey" is consistent with ZFC. In Section 6 we shall provide a third proof as a byproduct of the analysis of scales under PD.

Concerning Martin's Axiom MA, it is well-known that MA implies $\mathfrak{h}=2^{\aleph_{0}}$. Hence, by the facts mentioned above, $\mathrm{MA}+\neg \mathrm{CH}$ implies that all $\boldsymbol{\Sigma}_{2}^{1}$ sets have the classical Ramsey property.

A similar argument for the dualized case does not work: Brendle has shown in $\left[\right.$ Br00-2], that MA $+2^{\aleph_{0}}>\mathfrak{H}=\aleph_{1}$ is consistent with ZFC. However, this result does not preclude that $\mathrm{MA}+\neg \mathrm{CH}$ might imply that every $\Sigma_{2}^{1}$ set is dual Ramsey.

At the next level we like to mention the following

Fact 5.3. "Every $\boldsymbol{\Delta}_{3}^{1}$ set is dual Ramsey" is consistent with ZFC.

Proof. An $\omega_{1}$-iteration (or also an $\omega_{2}$-iteration) with countable support of dual Mathias forcing starting from $\mathbf{L}$ yields a model in which every $\boldsymbol{\Delta}_{3}^{1}$ set is dual Ramsey. The proof is exactly the same as the proof of the corresponding result for the classical Ramsey property given in [JuSh93]; the reason for this is that they needed only that Mathias forcing is proper and has the homogeneity property, but these two properties hold also for dual Mathias forcing.

The $\boldsymbol{\Delta}_{3}^{1}$ level is probably as far as we can get in ZFC without further assumptions. It is a famous open question whether the consistency of "Every $\boldsymbol{\Pi}_{3}^{1}$ set is Ramsey" implies the existence of an inner model with an inaccessible cardinal (cf. [Kan94, Question 11.16] \& [Rai84, p. 49]). Most likely, the dualized question is equally hard to conquer.

\section{Determinacy and the dual Ramsey property.}

We shall move on to arbitrary projective sets in this section. As we mentioned earlier, this means that we probably have to go beyond ZFC.

In [CaSi84, Section 5], the authors prove in fact that in the Solovay model constructed by collapsing an inaccessible cardinal to $\omega_{1}$ every projective set is dual Ramsey. As we remarked, it is unknown whether the inaccessible cardinal is necessary for that.

But there is another question connected to the dual Ramsey property of projective sets: As with the standard Ramsey property we can ask whether 
an appropriate amount of determinacy implies the dual Ramsey property. As usually with regularity properties of sets of reals we would expect that $\operatorname{Det}\left(\boldsymbol{\Pi}_{n}^{1}\right)$ implies the dual Ramsey property for all $\boldsymbol{\Sigma}_{n+1}^{1}$ sets. But a direct implication using determinacy is not as easy as with the more prominent regularity properties (as Lebesgue measurability and the Baire property) since the games connected to the dual Ramsey property (the Banach-Mazur games in the dual Ellentuck topology) cannot be played using natural numbers.

The same problem had been encountered with the standard Ramsey property and had been solved in [HarKe81] by making use of the scale property and the Periodicity Theorems 2.8 and 2.9:

Theorem 6.1. If $\operatorname{Det}\left(\boldsymbol{\Delta}_{2 n+2}^{1}\right)$, then every $\boldsymbol{\Pi}_{2 n+2}^{1}$ set is Ramsey.

The main ingredient of this proof was an analysis of the models $\mathbf{L}\left[T_{2 n+1}\right]$ under Determinacy assumptions (Lemma 6.3). In the following we shall give a brief review of the result with sketches of an adaptation to our context.

Lemma 6.2. Let $\Gamma$ be any $\omega$-parametrized pointclass. If $U$ is $\omega$-universal for $\Gamma, A \in \Gamma, \mathbf{N}$ any model, and $T \in \mathbf{N}$ a tree such that $p[T]=U$. Then there is a tree $S \in \mathbf{N}$ such that $p[S]=A$.

Proof. This is basically [Kan94, Proposition 13.13 (g)], apart from the assertion that $S \in \mathbf{N}$. But this is clear since the reduction function reducing $A$ to $U$ is just the trivial function $x \mapsto\left\langle n_{0}, x\right\rangle$ (where $n_{0}$ is the index of $A$ in $U$ ) and hence in $\mathbf{N}$.

Lemma 6.3. Assume $\operatorname{Det}\left(\boldsymbol{\Delta}_{2 n+2}^{1}\right)$. Then $[\omega]^{\omega} \cap \mathbf{L}\left[T_{2 n+1}\right]=C_{2 n+2}$. In particular, this is a countable set.

Proof Sketch. We shall very roughly sketch the argument of [HarKe81, Theorem 7.2.1]:

First of all $[\omega]^{\omega} \cap \mathbf{L}\left[T_{2 n+1}\right]$ is easily seen to be $\Sigma_{2 n+2}^{1}$. That every countable $\Sigma_{2 n+2}^{1}$ set of reals is a member of $\mathbf{L}\left[T_{2 n+1}\right]$ follows directly from Mansfield's Theorem (cf. [Kan94, Theorem 14.7]) and Lemma 6.2. ${ }^{16}$

So, what is left to show is that $[\omega]^{\omega} \cap \mathbf{L}\left[T_{2 n+1}\right]$ actually is countable. The proof uses the following steps:

(i) Fix a $\Pi_{2 n+1}^{1}$ norm $\varphi^{*}: P_{2 n+1} \rightarrow \delta_{2 n+1}^{1}$ which exists according to Theorem 2.8.

(ii) Using $\varphi^{*}$, code the tree $T_{2 n+1}$ by some $A \subseteq \boldsymbol{\delta}_{2 n+1}^{1}$ and show that $\mathbf{L}\left[T_{2 n+1}\right]=\mathbf{L}[A]$.

(iii) For arbitrary subsets $X \subseteq \delta_{2 n+1}^{1}$, define $X^{*}:=\left\{z \in[\omega]^{\omega}: \varphi^{*}(z) \in\right.$ $X\}$.

${ }^{16}$ Note that by Theorem 2.10 the choice of the complete set for the definition of the $\mathbf{L}\left[T_{2 n+1}\right]$ doesn't matter. 
(iv) Show: If $X^{*} \in \Delta_{2 n+2}^{1}$, then the set $[\omega]^{\omega} \cap \mathbf{L}[X]$ is contained in a countable $\Sigma_{2 n+2}^{1}$ set.

(v) Compute: $A^{*} \in \Delta_{2 n+2}^{1}$.

Harrington and Kechris used this result to receive results about projective sets from PD alone that formerly could only be derived from stronger hypotheses. The results for the classical Ramsey property follows Solovay's argument for the $\boldsymbol{\Sigma}_{2}^{1}$ case. We shall outline this argument in full generality and then apply it to the dual Ramsey property.

At first we need to relativize Lemma 6.3 in two different parameters:

Lemma 6.4. Assume $\operatorname{Det}\left(\boldsymbol{\Delta}_{2 n+2}^{1}\right)$. Let $x, y \in[\omega]^{\omega}$ be any real numbers. Then $[\omega]^{\omega} \cap \mathbf{L}\left[T_{2 n+1}^{y}, x\right]=C_{2 n+2}(x \oplus y)$.

Proof Sketch. As an immediate relativization of of Lemma 6.3 (for the pointclass $\Pi_{2 n+1}^{1}(y)$ instead of $\left.\Pi_{2 n+1}^{1}\right)$, we get:

$$
[\omega]^{\omega} \cap \mathbf{L}\left[T_{2 n+1}^{y}\right]=C_{2 n+2}(y) .
$$

To show that $[\omega]^{\omega} \cap \mathbf{L}\left[T_{2 n+1}^{y}, x\right]$ is a countable set, we have to relativize (iv) and (v) again. The obvious relativization of (iv) is

(iv*) If $X^{*} \in \Delta_{2 n+2}^{1}(x \oplus y)$, then the set $[\omega]^{\omega} \cap \mathbf{L}[X]$ is contained in a countable $\Sigma_{2 n+2}^{1}(x \oplus y)$ set.

Since $\mathbf{L}\left[T_{2 n+1}^{y}\right]=\mathbf{L}[A]$ for some $A \subseteq \boldsymbol{\delta}_{2 n+1}^{1}$ such that $A^{*}$ is $\Delta_{2 n+2}^{1}(y)$ according to (ii) and (v), we know that $\mathbf{L}\left[T_{2 n+1}^{y}, x\right]=\mathbf{L}[A, x]$. Thus we have to find a set $B \subseteq \boldsymbol{\delta}_{2 n+1}^{1}$ such that $\mathbf{L}[B]=\mathbf{L}[A, x]$ and $B^{*} \in \Delta_{2 n+1}^{1}(x \oplus y)$. This would prove the theorem.

The natural choice for $B$ is:

$$
\begin{aligned}
B:= & \left\{\alpha \in \delta_{2 n+1}^{1}:(\alpha \geq \omega \wedge \alpha \in A)\right. \text { or } \\
& (\alpha<\omega \wedge \exists n(\alpha=2 n \wedge n \in A)) \text { or } \\
& (\alpha<\omega \wedge \exists n(\alpha=2 n+1 \wedge n \in x))\} .
\end{aligned}
$$

Obviously, $B \in \mathbf{L}[A, x]$ and $A$ and $x \in \mathbf{L}[B]$, so $\mathbf{L}[A, x]=\mathbf{L}[B]$. Thus, what is left is to show that $B^{*}$ is $\Delta_{2 n+2}^{1}(x \oplus y)$. But this is easy to see using the definition of $B$, and the facts that $\varphi^{*}$ was a $\Pi_{2 n+1}^{1}$ norm and that $A^{*}$ was $\Delta_{2 n+2}^{1}(y)$.

The following lemma is an obvious generalization of Shoenfield's Absoluteness Lemma (cf. also [Mo80, Theorem 8G.10]):

Lemma 6.5. $\Sigma_{2 n+2}^{1}(x)$ formulae are absolute for models containing $T_{2 n+1}^{x}$, i.e., if $\mathbf{N}$ is a model with $T_{2 n+1}^{x} \in \mathbf{N}$ and $\varphi$ is a $\Sigma_{2 n+2}^{1}$ formula, then

$$
\forall X \in \mathbf{N}(\mathbf{N} \models \varphi[X, x] \Longleftrightarrow \mathbf{V} \models \varphi[X, x]) \text {. }
$$


Proof. By Theorem 2.10, we can assume that $T_{2 n+1}^{x}$ was constructed using an $\omega$-universal set for $\Pi_{2 n+1}^{1}(x)$, enabling us to use Lemma 6.2.

Thus every $\Pi_{2 n+1}^{1}(x)$ set is represented by a tree $S \in \mathbf{N}$. We easily get a tree $S^{*}$ for each $\Sigma_{2 n+2}^{1}(x)$ set (cf. [Kan94, Proposition $\left.13.13(\mathrm{~d})\right]$ ).

But now the theorem follows from standard absoluteness of illfoundedness as in Shoenfield's proof (cf. [Kan94, Exercise 12.9 (a)]).

Theorem 6.6. Let $x \in[\omega]^{\omega}$ be a real. Suppose that there is a dual Mathias generic partition over $\mathbf{L}\left[T_{2 n+1}^{x}\right]$. Then every $\Sigma_{2 n+2}^{1}(x)$ set is dual Ramsey.

Proof. Let $A$ be a $\Sigma_{2 n+2}^{1}(x)$ set and $\varphi$ a $\Sigma_{2 n+2}^{1}(x)$ expression describing $A$, i.e.,

$$
\forall y(y \in A \Longleftrightarrow \varphi[y]) \text {. }
$$

By Fact 2.1 we find a $\mathbb{M}^{\star}$-condition $\langle\emptyset, X\rangle \in \mathbf{L}\left[T_{2 n+1}^{x}\right]$ such that

$$
\text { either }\langle\emptyset, X\rangle \Vdash \varphi\left(\operatorname{pc}\left(\boldsymbol{X}_{G}\right)\right) \text { or }\langle\emptyset, X\rangle \Vdash \neg \varphi\left(\operatorname{pc}\left(\boldsymbol{X}_{G}\right)\right) \text {, }
$$

where $\boldsymbol{X}_{G}$ is the name for a dual Mathias generic partition.

Without loss of generality, we assume the former. By our assumption, we actually have a generic partition $Z$ over $\mathbf{L}\left[T_{2 n+1}^{x}\right]$ with $\langle\emptyset, X\rangle \in G_{Z}$, where $G_{Z}$ is the filter associated to $Z$, i.e.,

$$
\langle t, Y\rangle \in G_{Z} \Longleftrightarrow Z \in(t, Y)^{\omega} .
$$

This means that $Z \in(X)^{\omega}$. Now by 2.1 (homogeneity of $\mathbb{M}^{\star}$ ) again, every element $Z^{*}$ of $(Z)^{\omega}$ is also $\mathbb{M}^{\star}$-generic over $\mathbf{L}\left[T_{2 n+1}^{x}\right]$. Since $G_{Z^{*}} \subseteq G_{Z}$, we still have $\langle\emptyset, X\rangle \in G_{Z^{*}}$. Consequently, we have $\mathbf{L}\left[T_{2 n+1}^{x}\right]\left[Z^{*}\right] \models \varphi\left[\operatorname{pc}\left(Z^{*}\right)\right]$. But $\varphi$ was absolute for models containing $T_{2 n+1}^{x}$ by Lemma 6.5 , hence we have $\mathbf{V}=\varphi\left[\operatorname{pc}\left(Z^{*}\right)\right]$.

Summing up, we have found a partition $Z$ such that $\left\{\operatorname{pc}\left(Z^{*}\right): Z^{*} \sqsubseteq Z\right\} \subseteq$ $A$. This is exactly what we had to show by Observation 2.5.

Note that this type of argument probably will not work if you replace "dual Ramsey" by "completely dual Ramsey". What you would have to do is to relativize the argument to arbitrary partitions $W \in \mathbf{V}$. But at least this does not work in the classical case: Brendle has shown in [Br00-1] that in any model containing one Mathias real over a ground model $\mathbf{N}$, there is an Ellentuck neighbourhood that doesn't contain any Mathias reals over $\mathbf{N}$.

Another useful comment about Theorem 6.6 is that if you look at the case $n=0$ you get a third proof of the consistency of "Every $\boldsymbol{\Sigma}_{2}^{1}$ set is dual Ramsey":

Corollary 6.7. Suppose that for each real $x \in[\omega]^{\omega}$ there is a dual Mathias generic partition over $\mathbf{L}[x]$. Then every $\boldsymbol{\Sigma}_{2}^{1}$ set is dual Ramsey.

Proof. Immediate from Theorem 6.6, keeping in mind that $T_{1} \in \mathbf{L}$ by [KeMo78, 9C], as mentioned in Subsection 2.7. 
This particularly generic version of proving the consistency of properties of $\boldsymbol{\Sigma}_{2}^{1}$ sets should be compared to analogous results for Random forcing, Cohen forcing and Hechler forcing. ${ }^{17}$

We now move on to use Lemma 6.4 and Theorem 6.6 to get that Determinacy implies the dual Ramsey property:

Corollary 6.8. Assume $\operatorname{Det}\left(\boldsymbol{\Delta}_{2 n+2}^{1}\right)$. Then every $\boldsymbol{\Sigma}_{2 n+2}^{1}$ set is dual Ramsey.

Proof. By Lemma 6.4, we get that for any reals $x, y \in[\omega]^{\omega}$, the set of reals in $\mathbf{L}\left[T_{2 n+1}^{x}, y\right]$ is countable.

By the same argument that is used to show that $\forall x\left(\aleph_{1}^{\mathbf{L}[x]}<\aleph_{1}^{\mathbf{V}}\right)$ implies that $\aleph_{1}^{\mathbf{V}}$ is strongly inaccessible in every $\mathbf{L}[x]$, we get that $\mathcal{P}\left((\omega)^{\omega}\right) \cap$ $\mathbf{L}\left[T_{2 n+1}^{x}, y\right]$ is countable for arbitrary choices of $x$ and $y \in[\omega]^{\omega}$.

Thus there are dual Mathias generic partitions over each $\mathbf{L}\left[T_{2 n+1}^{x}, y\right]$, in particular over each $\mathbf{L}\left[T_{2 n+1}^{x}\right]$, and we can use Theorem 6.6 to prove the claim.

\section{Appendix: Game-filters have the segment-colouring-property.}

Let $\mathfrak{F} \subseteq(\omega)^{\omega}$ be an ultrafilter. Associated with $\mathfrak{F}$ we define the game $\mathcal{G}_{\mathfrak{F}}$ as follows. This type of game, which is the Choquet-game with respect to the dual Ellentuck topology (cf. [Ke95, 8.C]), was first suggested by Kastanas in $[$ Kas83].
I
$\left\langle t_{0}, Y_{0}\right\rangle$
$\left\langle t_{1}, Y_{1}\right\rangle$
$\left\langle t_{2}, Y_{2}\right\rangle$

II

$\left\langle X_{0}\right\rangle$

$\left\langle X_{1}\right\rangle$

$\left\langle X_{2}\right\rangle$

All the moves $X_{n}$ of player II must be elements of the ultrafilter $\mathfrak{F}$ and all the moves $\left\langle t_{n}, Y_{n}\right\rangle$ of player I plays must be such that $Y_{n} \in \mathfrak{F}$ and $\left(t_{n}^{*}, Y_{n}\right)^{\omega}$ is a dual Ellentuck neighbourhood. Further, the $n$th move $X_{n}$ of player II is such that $X_{n} \in\left(t_{n}^{*}, Y_{n}\right)^{\omega}$ and then player I plays $t_{n+1}$ such that $t_{n}^{*} \preccurlyeq t_{n+1}^{*} \sqsubseteq$ $X_{n}$ and $\left|t_{n+1}\right|=\left|t_{n}\right|+1=\left|t_{0}\right|+n+1$. Player I wins if and only if the unique $Y$ with $t_{n} \preccurlyeq Y$ (for all $n$ ) is not in $\mathfrak{F}$.

An ultrafilter $\mathfrak{F}$ is a game filter if and only if player I has no winning strategy in the game $\mathcal{G}_{\mathfrak{F}} \cdot{ }^{18}$

In the following we outline the proof that game filters are also scp-filters. The crucial point will be to show the Preliminary Lemma 7.1, which is in

\footnotetext{
${ }^{17} \mathrm{Cf}$. [BrLö99]. Note that in most cases the existence of generics doesn't give more than regularity at the $\boldsymbol{\Delta}_{2}^{1}$ level, and something more than mere existence is needed for the $\boldsymbol{\Sigma}_{2}^{1}$ level.

${ }^{18}$ For the existence of game filters see [Halb98-1], where one can find also some results concerning dual Mathias forcing restricted to such filters.
} 
fact Carlson's Lemma (cf. [CaSi84, Lemma 2.4]) restricted to game filters. But first we have to give some notations.

Let $s, t \in(\mathbb{N})$ be such that $s \sqsubseteq t,|s|=n$ and $|t|=m$. For $k$ with $k \leq m-n$ let

$$
(t)_{s}^{k}:=\{u \in(\mathbb{N}): \operatorname{dom}(u)=\operatorname{dom}(t) \wedge s \preccurlyeq u \sqsubseteq t \wedge|u|=|s|+k\} .
$$

For $s \preccurlyeq t \sqsubseteq X$, let

$$
(t, X)_{s}^{k *}:=\left\{u \in(\mathbb{N}): t \preccurlyeq u^{*} \sqsubseteq X \wedge|u|=|s|+k\right\},
$$

and let $(X)_{s}^{k *}:=(s, X)^{(n+k) *}$.

We have chosen this notation following [CaSi84, Definition 2.1], where one can consider $s$ as an alphabet of cardinality $n$.

For the remainder of this section, let $\mathfrak{F}$ be an arbitrary but fixed game filter.

Preliminary Lemma 7.1. Let $s \preccurlyeq X \in \mathfrak{F}$ and $\pi:(X)_{s}^{0 *} \rightarrow l$, then there exists a $Y \in(s, X)^{\omega} \cap \mathfrak{F}$ such that $\pi \uparrow(Y)_{s}^{0 *}$ is constant.

Following the ideas of the proof of Theorem 6.3 of [CaSi84], the proof of the Preliminary Lemma will be given in a sequence of lemmas. We start by stating the well-known Hales--Jewett Theorem in our notation.

Hales-Jewett Theorem 7.2. Let $s \in(\mathbb{N})$. For all $d \in \omega$, there is an $h \in \omega$ such that for any $t \in(\mathbb{N})$ with $s \preccurlyeq t$ and $|t|=|s|+h$, and for any colouring $\tau:(t)_{s}^{0} \rightarrow d$, there is a $u \in(t)_{s}^{1}$ such that $(u)_{s}^{0}$ is monochromatic.

The number $h$ in the Hales-Jewett Theorem depends only on the number $d$ and the size of $|s|$. Let $\operatorname{HJ}(d,|s|)$ denote the smallest number $h$ which verifies the Hales-Jewett Theorem.

Let $s, t \in(\mathbb{N})$ and $X \in \mathfrak{F}$ be such that $s \preccurlyeq t \sqsubseteq X$. A set $K \subseteq(\mathbb{N})$ is called dense in $(t, X)^{\omega}$, if for all $Y \in(t, X)^{\omega} \cap \mathfrak{F}$, there is a $u$ with $t \preccurlyeq u^{*} \sqsubseteq Y$ which belongs to $K$. A set $D \subseteq(\omega)_{s}^{k *}$ is called $k$-dense in $(t, X)_{s}^{k *}$, if for all $Y \in(t, X)^{\omega} \cap \mathfrak{F}$, we have $(t, Y)_{s}^{k *} \cap D \neq \emptyset$.

Lemma 7.3. Let $s \preccurlyeq t \preccurlyeq X \in \mathfrak{F}$ and assume that $D \subseteq(\omega)_{s}^{0 *}$ is 0 -dense in $(X)_{s}^{0 *}$. Further assume that $K=\left\{u: t \preccurlyeq u \wedge(u)_{s}^{0} \cap D \neq \emptyset\right\}$ is dense in some $(t, Z)^{\omega}$, where $Z \in(t, X)^{\omega} \cap \mathfrak{F}$. Then there is an $\bar{s}^{*} \sqsubseteq Z$ with $t \preccurlyeq \bar{s}$ such that for all $v^{*} \sqsubseteq Z$ with $\bar{s} \preccurlyeq v$ we have $(v)_{s}^{0} \cap D \neq \emptyset$.

Proof. We shall define a strategy for player I in the game $\mathcal{G}_{\mathfrak{F}}$, such that player I can follow this strategy just in the case when Lemma 7.3 fails. This means that for every $\bar{s}^{*} \sqsubseteq Z$ with $t \preccurlyeq \bar{s}$ there is a $v^{*} \sqsubseteq Z$ with $\bar{s} \preccurlyeq v$ such that $(v)_{s}^{0} \cap D=\emptyset$.

Let $t_{0}^{*} \sqsubseteq Z$ be such that $t \preccurlyeq t_{0},|t|=\left|t_{0}\right|$ and $\left(t_{0}\right)_{s}^{0} \cap D=\emptyset$. Further put $Y_{0}=t_{0} \sqcap Z$ and player I plays $\left\langle t_{0}, Y_{0}\right\rangle$. Assume $\left\langle t_{m}, Y_{m}\right\rangle$ is the $m$ th move of player I and player II replies with $\left\langle X_{m}\right\rangle$. If the lemma fails with $\bar{s}=t_{m}^{*}$, 
player I can play $\left\langle t_{m+1}, Y_{m+1}\right\rangle$, according to the rules of the game, such that $\left(t_{m+1}\right)_{s}^{0} \cap D=\emptyset$.

Since $\mathfrak{F}$ is a game filter, the strategy of player I is not a winning strategy and the unique $Y \in(Z)^{\omega}$ such that $t_{m} \preccurlyeq Y$ (for all $m \in \omega$ ) belongs to $\mathfrak{F}$. Take an arbitrary $u$ with $t \preccurlyeq u^{*} \sqsubseteq Y$. For such a $u$ we find a $t_{n} \preccurlyeq Y$ such that $u \sqsubseteq t_{n}$ and $\operatorname{dom}(u)=\operatorname{dom}\left(t_{n}\right)$. By the strategy of player I we have $\left(t_{n}\right)_{s}^{0} \cap D=\emptyset$ and therefore $(u)_{s}^{0} \cap D=\emptyset$. But this is a contradiction to the assumption that $K$ is dense in $(t, Z)^{\omega}$. Hence, player I cannot follow this strategy, which completes the proof.

Lemma 7.4. Suppose $s \preccurlyeq t \preccurlyeq X \in \mathfrak{F}, D$ is 0 -dense in $(X)_{s}^{0 *}$ and $K=\{u$ : $\left.t \preccurlyeq u \wedge(u)_{s}^{0} \cap D \neq \emptyset\right\}$ is dense in $(t, Z)^{\omega}$, where $Z \in(t, X)^{\omega} \cap \mathfrak{F}$. Then there is $a \bar{t}^{*} \sqsubseteq Z$ with $t \preccurlyeq \bar{t}$ and $|\bar{t}|=|t|+1$ such that $(\bar{t})_{s}^{0} \subseteq D$.

Proof. Let $\bar{s}$ be as in the Lemma 7.3, and let $d:=\left|(\bar{s})_{s}^{0}\right|$. By the HalesJewett Theorem, let $h:=\operatorname{HJ}(d,|s|)$. Pick $v \in(\mathbb{N})$ such that $\bar{s} \preccurlyeq v^{*} \sqsubseteq X$ and $|v|=|\bar{s}|+h$. Let $\left\{s_{i}: s_{i} \in(\bar{s})_{s}^{0} \wedge i \in d\right\}$ be an enumeration of the elements of $(\bar{s})_{s}^{0}$. We colour $(v)_{s}^{0}$ by stipulating $\tau(u)=i$ if and only if $s_{i} \preccurlyeq u \wedge u \in D$. By the choice of $n$, there are $\bar{t} \in(v)_{s}^{1}$ such that $(\bar{t})_{s}^{0}$ is monochromatic, and therefore, $(\bar{t})_{s}^{0} \subseteq D$. Thus, we have found a $\bar{t}$ with $t \preccurlyeq \bar{t}$ and $|\bar{t}|=|t|+1$ such that $(\bar{t})_{s}^{0} \subseteq D$.

Lemma 7.5. Suppose $s \preccurlyeq X \in \mathfrak{F}$ and $D$ is 0 -dense in $(X)_{s}^{0 *}$. Then there are $t \in(X)_{s}^{1 *}$ and $Y \in(t, X)^{\omega} \cap \mathfrak{F}$ such that $\left\{u: t \preccurlyeq u \wedge(u)_{s}^{0} \subseteq D\right\}$ is 1-dense in $(t, Y)_{s}^{1 *}$.

Proof. In a similar way as above we can define a strategy for player I in the game $\mathcal{G}_{\mathfrak{F}}$, such that player I can follow this strategy only if Lemma 7.4 fails. But if Lemma 7.4 is wrong, this would yield - because $\mathfrak{F}$ is a game filter - a contradiction (cf. [CaSi84, Lemma 6.5]).

Notice that in Lemma 7.5 we did not require that the $r \in(\mathbb{N})$ for which we have $r^{*} \preccurlyeq t \in(X)_{s}^{1 *}$ belongs to $D$. This we do in

Lemma 7.6. Suppose $s \preccurlyeq X \in \mathfrak{F}$ and $D$ is 0 -dense in $(X)_{s}^{0 *}$. Then there are $r^{*} \preccurlyeq t \in(X)_{s}^{1 *}$ and $Y \in(t, X)^{\omega} \cap \mathfrak{F}$ such that $\left\{u: t \preccurlyeq u \wedge(u)_{s}^{0} \subseteq D\right\}$ is 1 -dense in $(t, Y)_{s}^{1 *}$ and $r \in D$.

Proof. Let $Y_{0} \in\left(t_{0}, X\right)^{\omega} \cap \mathfrak{F}$ be as in the conclusion of Lemma 7.5. Thus $D_{0}:=\left\{u: t_{0} \preccurlyeq u \wedge(u)_{s}^{0} \subseteq D\right\}$ is 1-dense in $\left(t_{0}, Y_{0}\right)_{s}^{1 *}$. Using Lemma 7.5, player I can play $\left\langle t_{m}, Y_{m}\right\rangle$ at the $m$ th move such that $D_{m}:=\left\{u: t_{m} \preccurlyeq\right.$ $\left.u \wedge(u)_{s}^{0} \subseteq D\right\}$ is $(m+1)$-dense in $\left(t_{m}, Y_{m}\right)_{s}^{(m+1) *}$.

Because player I has no winning strategy, the unique $Y \in(s, X)^{\omega}$ such that $t_{m} \preccurlyeq Y$ (for all $m$ ) belongs to $\mathfrak{F}$, and because $D$ is 0 -dense in $(X)_{s}^{0 *}$, there is an $r \in(Y)_{s}^{0 *}$ which belongs to $D$. Let $\bar{t} \in\left(t_{m}\right)_{s}^{1}$ be such that $r^{*} \preccurlyeq \bar{t}$. Since $(\bar{t})_{s}^{0} \subseteq\left(t_{m}\right)_{s}^{0}$ and because $D_{m}$ is $(m+1)$-dense in $\left(t_{m}, Y_{m}\right)_{s}^{(m+1) *}$ we 
get $\left\{u: \bar{t} \preccurlyeq u \wedge(u)_{s}^{0} \subseteq D\right\}$ is 1-dense in $\left(\bar{t}, Y_{m}\right)_{s}^{1 *}$. Hence, we have found an $r^{*}$ such that $\left\{u: r^{*} \preccurlyeq u \wedge(u)_{s}^{0} \subseteq D\right\}$ is 1-dense in $\left(r^{*}, Y\right)_{s}^{1 *}$ and $r \in D$.

Now we can go back to the

Proof of the Preliminary Lemma. Let $s \preccurlyeq X \in \mathfrak{F}$. We have to show that for any colouring $\pi:(X)_{s}^{0 *} \rightarrow l$, there is a $Y \in(s, X)^{\omega} \cap \mathfrak{F}$ such that $(Y)_{s}^{0 *}$ is monochromatic.

It is easy to see that at least one of the colours is 0 -dense in $(X)_{s}^{0 *}$, say $j$ and let $D:=\left\{t \in(X)_{s}^{0 *}: \pi(t)=j\right\}$. Now we can prove the Preliminary Lemma in almost the same way as Lemma 7.6, the only difference is that player I uses now Lemma 7.6 to construct the $m$ th move, instead of Lemma 7.5.

Finally we get the main result of this section.

Proposition 7.7. Each game filter is also an scp-filter.

Proof. We have to show that for any colouring $\pi:(s, \omega)^{(|s|+k) *} \rightarrow r$, where $r$ and $k$ are positive natural numbers and $s \in(\mathbb{N})$, there is an $X \in \mathfrak{F}$ such that $s \preccurlyeq X$ and $(s, X)^{(|s|+k) *}$ is monochromatic.

Following the proof of [Halb $\infty$, Theorem] and using the Preliminary Lemma, it is not hard to define a strategy for player I in such a way that if player I follows this strategy, then for the resulting partition $X$-which must belong to $\mathfrak{F}$, since $\mathfrak{F}$ is a game filter - we get $s \preccurlyeq X$ and $(s, X)^{(|s|+k) *}$ is monochromatic.

We have seen that every game filter has the segment-colouring property. It seems that the reverse implication is unlikely, since a strategy for player I cannot be encoded by a real number, which makes it hard (if not impossible) to prove that $\mathrm{CH}$ implies the existence of game filters. But on the other hand we know that scp-filters always exist if we assume $\mathrm{CH}$.

\section{References}

[AdMo68] J.W. Addison and Y.N. Moschovakis, Some consequences of the axiom of definable determinateness, Proceedings of the National Academy of Sciences U.S.A., 59 (1968), 708-712, MR 36 \#4979, Zbl 186.25302.

[BaJu95] T. Bartoszyński and H. Judah, Set Theory: On the Structure of the Real Line, A. K. Peters, Wellesley, 1995, MR 96k:03002, Zbl 834.04001.

[BeKe84] H.S. Becker and A.S. Kechris, Sets of ordinals constructible from trees and the third Victoria Delfino Problem, in 'Axiomatic Set Theory' (J.E. Baumgartner, D.A. Martin, S. Shelah, Eds.), Contemporary Mathematics, 31, American Mathematical Society, (1984), 13-29, MR 86a:03051, Zbl 629.03027. 
[Br00-1] J. Brendle, How small can the set of generics be?, in 'Logic Colloquium '98, Proceedings of the 1998 Association for Symbolic Logic European Summer Meeting, Prague, Czech Republic, 1998' (S.R. Buss, P. Hájek, P. Pudlák, Eds.), Lecture Notes in Logic, 13, Springer, (2000), 92-109, MR 2001a:03099.

[Br00-2] , Martin's axiom and the dual distributivity number, Mathematical Logic Quarterly, 46(2) (2000), 241-248, MR 2001c:03084.

[BrLö99] J. Brendle and B. Löwe, Solovay-type characterizations of forcing algebras, Journal of Symbolic Logic, 64 (1999), 1307-1323, MR 1,779764, Zbl 945.03071.

[CaSi84] T.J. Carlson and S.G. Simpson, A dual form of Ramsey's Theorem, Advances in Mathematics, 53 (1984), 265-290, MR 85h:04002, Zbl 564.05005.

[El74] E. Ellentuck, A new proof that analytic sets are Ramsey, Journal of Symbolic Logic, 39 (1974), 163-165, MR 50 \#1887, Zbl 292.02054.

[Halb98-1] L. Halbeisen, Symmetries between two Ramsey poperties, Archive for Mathematical Logic, 37 (1998), 241-260, MR 99i:03055, Zbl 937.03057.

[Halb98-2] _ On shattering, splitting and reaping partitions, Mathematical Logic Quarterly, 44 (1998), 123-134, MR 98k:03102, Zbl 896.03036.

[Halb $\infty] \quad$ A Ramsey type theorem and its corollaries, preprint.

[HalbJu96] L. Halbeisen and H. Judah, Mathias absoluteness and the Ramsey property, Journal of Symbolic Logic, 61 (1996), 177-193, MR 97c:03119, Zbl 851.03013.

[HalbLö $\infty$ L. Halbeisen and B. Löwe, Ultrafilter spaces on the semilattice of partitions, Topology and its Applications, to appear.

[HalJe63] A.W. Hales and R.I. Jewett, Regularity and positional games, Transactions of the American Mathematical Society, 106 (1963), 222-229, MR 26 \#1265, Zbl 113.14802.

[HarKe81] L.A. Harrington and A.S. Kechris, On the determinacy of games on ordinals, Annals of Mathematical Logic, 20 (1981), 109-154, MR 83c:03044, Zbl 489.03018. Zbl 419.03028.

[JuSh89] H. Judah and S. Shelah, $\Delta_{2}^{1}$-sets of reals, Annals of Pure and Applied Logic, 42 (1989), 207-223.

[JuSh93] _ _ $\quad \Delta_{3}^{1}$-sets of reals, Journal of Symbolic Logic, 58 (1993), 72-80, MR 94c:03067, Zbl 786.03036.

[Kan94] A. Kanamori, The Higher Infinite, Perspectives in Mathematical Logic, Springer-Verlag, Berlin Heidelberg, 1994, MR 96k:03125, Zbl 813.03034.

[Kas83] I.G. Kastanas, On the Ramsey property for sets of reals, Journal of Symbolic Logic, 48 (1983), 1035-1045, MR 85j:03080, Zbl 527.90101.

[Ke95] A.S. Kechris, Classical Descriptive Set Theory, Graduate Texts in Mathematics, 156, Springer-Verlag, New York, 1995, MR 96e:03057, Zbl 819.04002.

[KeMo72] A.S. Kechris and Y.N. Moschovakis, Two Theorems about Projective Sets, Israel Journal of Mathematics, 12 (1972), 391-399, MR 48 \#1900, Zbl 257.02034. 
[KeMo78] _ Notes on the Theory of Scales, in 'Cabal Seminar 76-77', Proceedings, Caltech-UCLA Logic Seminar, 1976-77 (A.S. Kechris and Y.N. Moschovakis, Eds.), Lecture Notes in Mathematics, 689, SpringerVerlag, Berlin, (1978), 1-53, MR 83b:03059, Zbl 397.032.

[Ku83] K. Kunen, Set Theory, an Introduction to Independence Proofs, Studies in Logic and the Foundations of Mathematics, 102, North Holland, Amsterdam, 1983, Zbl 534.03026.

[Mar68] D.A. Martin, The axiom of determinateness and reduction principles in the analytic hierarchy, Bulletin of the American Mathematical Society, 74 (1968), 687-689, MR 37 \#2607, Zbl 165.31605.

[Mat77] A.R.D. Mathias, Happy families Annals of Mathematical Logic, 12 (1977) 59-111, MR 58 \#10462, Zbl 369.02041.

[Mo71] Y.N. Moschovakis, Uniformization in a playful universe, Bulletin of the American Mathematical Society, 77 (1971), 731-736, MR 44 \#2609, Zbl 232.04002.

[Mo80] _ Descriptive Set Theory Studies in Logic and the Foundations of Mathematics, 100, North-Holland, Amsterdam, 1980, MR 82e:03002, Zbl 433.03025.

[Pl86] S. Plewik, On completely Ramsey sets, Fundamenta Mathematicae, 127 (1986), 127-132, MR 88d:04005.

[Rai84] J. Raisonnier, A mathematical proof of S. Shelah's Theorem on the Measure Problem and related results, Israel Journal of Mathematics, 48 (1984), 48-56, MR 86g:03082b, Zbl 596.03056.

[Ram29] F.P. Ramsey, On a problem of formal logic, Proceedings of the London Mathematical Society, Ser. II, 30 (1929), 264-286.

[St95] J.R. Steel, Projectively well-ordered inner models, Annals of Pure and Applied Logic, 74 (1995), 77-104, MR 96h:03089, Zbl 821.03023.

Received September 14, 1999. The first author wishes to thank the Swiss National Science Foundation for its support during the time at U.C. Berkeley in which the research for this paper has been done. The second author wishes to thank the Studienstiftung des deutschen Volkes for the funding of his travels to Boise ID and Las Vegas NV, and in particular Gunter Fuchs (Berlin).

QueEn's UNIVERSity BelFAsT

BELFAST BT7 1NN

NORTHERN IRELAND

E-mail address: halbeis@qub.ac.uk

Mathematisches Institut

Rheinische Friedrich-Wilhelms-UniversitätT BonN

Beringstrasse 6

53115 BONN

Germany

E-mail address: loewe@math.uni-bonn.de 